\title{
Accelerated Chip-Level Thermal Analysis Using Multilayer Green's Function
}

\author{
Baohua Wang and Pinaki Mazumder, Fellow, IEEE
}

\begin{abstract}
Continual scaling of transistors and interconnects has exacerbated the power and thermal management problems in the design of ultralarge-scale integrated (ULSI) circuits. This paper presents an efficient thermal-analysis method of $\mathcal{O}(N \lg N)$ complexity, where $N$ is the number of blocks that discretize the heat-source or temperature-observation regions. The method is named LOTAGre and formulated using the Green's function for heat conduction through multiple-layer materials, which account for the structure of ULSI chips and the accompanying heat sinks and mounting accessories. In addition to analyzing the thermal effects of the distributive heat sources, LOTAGre also considers the ambient temperature effects that are generally excluded in conventional Green's function-based thermal-analysis tools in order to avoid the concomitant analytical complexity. By employing the well-known eigen-expansion technique and classical transmissionline theory, fully analytical and explicit formulas are derived in this paper for the multilayer Green's function with the inclusion of the $s$-domain version, the homogeneous and inhomogeneous solutions to the heat-conduction equation. Then, the discrete cosine transform and its inversion are employed to accelerate the numerical computation of the homogeneous and inhomogeneous solutions. This paper includes extensive experimental results to demonstrate that LOTAGre can be as accurate as FLUENT, a sophisticated computational fluid dynamics tool, while speeding up the simulation run time by two to three orders of magnitude in comparison to FLUENT as well as conventional Green's functionbased thermal-analysis methods. This paper also discusses the limitations of using the traditional single-layer thermal model in thermal analysis for approximating a multilayer chip structure.
\end{abstract}

Index Terms-Algorithms, discrete cosine transforms, distributed parameter circuits, electrothermal effects, Green's function, temperature.

\section{INTRODUCTION}

$\mathbf{C}$ ONTINUAL shrinking of metal-oxide-semiconductor field-effect transistor (MOSFET) feature sizes and interconnect wire geometries profoundly affects the performance and reliability of ultralarge-scale integrated (ULSI) circuits. Accurate estimation of thermal profile and identification of localized hot spots inside a chip are utterly important in verifying and predicting the performance and reliability of the microelectronic chip before its actual fabrication. In the stateof-the-art sub-100 nanometer complementary MOS (CMOS) process technologies with hundreds of million transistors

Manuscript received December 8, 2005; revised March 17, 2006 and May 3, 2006. This work was supported in part by a MURI grant. This paper was recommended by Associate Editor L. M. Silveira.

The authors are with the Department of Electrical Engineering and Computer Science, University of Michigan, Ann Arbor, MI 48109-2121 USA (e-mail: baohuaw@eecs.umich.edu; mazum@eecs.umich.edu).

Digital Object Identifier 10.1109/TCAD.2006.883919 packed densely inside a chip, precise modeling of heat generation and the concomitant steady-state thermal distribution across the die of a chip has become crucial since the ULSI chips are operating at several gigahertz $(\mathrm{GHz})$ of clock speed. In addition to causing catastrophic thermal rundown, high chip temperature may exacerbate many types of subtle timing failures. For example, the MOSFET carrier mobility and threshold voltage reduce at a higher gate temperature. This temperatureinduced lowering of MOSFET parameters may lead to anomalous timing behavior of a logic gate. As corroborated by the alpha-power law MOSFET model [1], [2] it may decrease the gate delay if the gate supply voltage is higher than the zerotemperature coefficient (ZTC) point, e.g., $1.2 \mathrm{~V}$, while it may increase the gate delay if the gate supply voltage is below the ZTC point. Gate temperature also affects the gate subthreshold leakage current and thereby its static power consumption. Therefore, the temperature distribution within a chip must be calculated with high precision in order to ensure the robustness and the performance of a low-power and low-voltage integrated circuit (IC) operating in the subthreshold mode. It should be noted that with progressive IC supply voltage scaling toward $1 \mathrm{~V}$, the low-power IC design trend is moving toward the subthreshold mode of transistor operation [3], [4].

Temperature distribution inside a chip also prominently influences the signal propagation delays in on-chip global and local interconnects. Using low- $k$ dielectric constant materials in interconnects has considerably reduced the capacitive coupling between adjacent interconnects. However, it simultaneously reduces the material heat conductivities, thereby aggravating the interconnect self-Joule heating problem [5]. A higher interconnect temperature leads to a larger interconnect delay due to the increased interconnect resistance [6]. Thermal effects on gate and interconnect timing now pose major challenges to microprocessor designers in adjusting the clock distribution networks across the entire chip. Notably, the chip temperature gradients in lieu of a set of worst or best case chip temperature values must be considered to control the clock skews and to avoid synchronization failures under widely varying operating conditions of a chip [7]. On the other hand, the increased power density and the resulting elevation of temperature inside a chip will reduce the mean time to fail of metal wires due to accelerated electromigration of metal ions as described by the well-known Black's equation [8], [9]. To meet the stringent requirements of IC reliability concurrently with high performance of the IC, thermal-aware chip design has now become a part and parcel of chip design flow that incorporates repetitive full-chip thermal analysis over millions of transistors and interconnects [10]. 
In IC thermal analysis, the steady-state and time-dependent heat-conduction equations have been traditionally solved by grid-based methods, including the finite-difference (FD), the finite-element (FE), the boundary-element (BE), and the thermal network methods. Grid-based methods can expediently handle complicated chip geometries of a ULSI chip, albeit requiring large amounts of grids for volume/surface meshing. Therefore, various sophisticated acceleration techniques were introduced in grid-based thermal-analysis methods, such as the Krylov-subspace-based model order reduction technique, the alternating direction implicit method, the adaptive meshing technique, and sparse-matrix algorithms [11]-[13]. In comparison to grid-based methods, Green's function-based thermal-analysis methods are advantageous in ULSI physical design algorithms such as floor planning and cell placement [14]-[16]. Green's function-based methods do not discretize the chip regions without heat sources and temperature monitoring. Therefore, the Green's function-based methods improve simulation speed by a few orders of magnitude by selectively avoiding unnecessary and costly discretization of chip space. Using a simplified single-layer thermal model (STM), which considers one homogeneous material in the simulated environment, the heat-conduction Green's function under various boundary conditions (BCs) has been investigated in the literature and proposed for performing thermal analysis of ULSI chips [14], [16], [17]. Since a realistic chip comprises multiplelayer heterogeneous materials, the heat-conduction Green's function based on a multilayer thermal model (MTM) should have broad applications in IC thermal analysis, for example, in establishing compact thermal models and in analyzing the thermal distribution in the cutting-edge three-dimensional (3-D) ICs where multiple active layers are vertically integrated [18]-[20].

The Green's function used in the thermal analysis is derived from the Poisson's equation that widely occurs in extraction of parasitic elements within an IC. However, the adoption styles of the Green's function in thermal analysis and in parasitic exaction differ in several aspects. In parasitic exaction, charge sources are presumed to distribute through the surfaces of conducting geometries, thereby leading to the frequent use of a charge-sheet model [21]-[23]. In thermal analysis, however, heat sources span 3-D volumes of space. On the other hand, the chip horizontal dimensions are often approximated as infinite in parasitic extraction. The approximations permit one either to directly use the free-space Green's function or alternatively to utilize the radial symmetric property to simplify the Green's function by applying Hankel transformation [21], [24]. In thermal analysis, the real horizontal dimensions of the simulated chip should be used. Therefore, the specific BCs should be imposed on the four chip sidewalls to model heat insulation and other phenomena of heat transfer between the sidewalls and the ambient environment [16]. For substrate coupling analysis, although the Green's function was derived with the consideration of heterogeneous dielectric materials and chip sidewalls, zero potential and zero-potential gradient were assumed for the top and bottom surfaces of a chip [25]. Further, numerical stability problem was noticed in calculating the Green's function [25], [26]. However, in thermal analysis, general heat-convection
BCs should be imposed on the top and bottom surfaces of a chip.

For the aforementioned reasons, one objective of this paper is to derive the heat-conduction Green's function under various BCs for a multiple-layer structured chip consisting of heterogeneous heat-conduction materials. The other objective of this paper is to account for the effects of ambient temperatures, which have often been assumed uniformly surrounding the chip by traditional Green's function-based thermal-analysis methods [14]-[16]. Due to the existence of dissimilar temperature gradients at different boundaries of the chip and the inequity of heat flow from different chip surfaces to the outer environment, the ambient temperatures can be nonuniform. This paper introduces a Green's function-based thermal-analysis method which accurately accounts for ambient temperatures by separating the temperature distribution inside a chip into two parts: an inhomogeneous temperature distribution attributed to only the heat sources inside the chip and a homogeneous temperature distribution attributed to only the ambient temperatures around the chip.

The proposed method is of $\mathcal{O}(N \lg N)$ complexity in contrast to traditional Green's function-based thermal-analysis methods having much higher run-time complexities [14]-[16]. The traditional methods evaluate the chip temperature distribution according to the following procedure: Heat sources are at first modeled as discrete blocks; then, the temperatures of the observed chip regions are computed by summing the products of the heat-source power densities and the Green's function values. To evaluate the temperatures of $N$ target blocks raised by $N$ heat-source blocks, the procedure becomes equivalent to a direct matrix-vector product operation on an $N \times N$ matrix and an $N$-dimensional vector. Therefore, a thermalanalysis method of this type requires $\mathcal{O}\left(N^{2}\right)$ computations to obtain the intended temperatures. Such a quadratic increase of computational time becomes very prominent, especially when the method is used in an inner loop and iterated for many times. The proposed method in this paper avoids the dense matrix-vector product operation and significantly speeds up the thermal analysis of ULSI chips by utilizing the eigen-expansion technique based on orthogonal trigonometric functions.

The issue of thermal distribution in a rectangular multilayer structure has attracted intense research interest. The classic temperature solution for a three-layer structure with surface heat generation was given in [27], while in [28], a concise recursion relation for the surface temperature was presented without restricting the number of layers. To consider the surface heat convection and interior heat-generation issue, pseudolayer approximation and linear system formulation in frequency domain were proposed in [29]. In [30], a linear system formulation via the use of separation of variables was proposed to compute the eigenfunctions and time constants. In [31] and [32], twoport network was employed for handling thermal BCs. In [19], volume heat-generation issue was considered and transfer matrices were employed to describe the thermal transfer impedances. Most recently, in [33], general nonlinear heatconvection BCs were considered and a system of equations was suggested for handling heterogeneous materials. In this paper, analytical formulas for the multilayer heat-conduction Green's 
function including the $s$-domain version are presented, which not only can be used in the thermal analysis of ULSI chips, but also can be deployed in the thermal analysis of general multilayer structures and in the establishment of compact thermal models [19], [20]. For example, by using the derived multilayer Green's function, this paper obtains a surface-temperature solution that agrees with the recursion relation in [28]; and by using the $s$-domain Green's function, double Fourier series solution similar to that in [19] is obtained for the surface thermal transfer impedance. The eigen-expansion coefficients of the multilayer heat-conduction Green's function, including the $s$-domain version, are given by fully analytical and explicit formulas, thereby providing a means for fast thermal characterization of multilayer structures. Additionally, this paper employs two-dimensional (2-D) discrete cosine transform (DCT) and inverse DCT in computing the double Fourier series.

The rest of this paper is organized as follows. Section II mathematically describes the chip-level thermal-analysis issue. Section III introduces the theoretical framework for applying the Green's function in thermal analysis. Section IV derives the inhomogeneous solution. Specifically, the multilayer heatconduction Green's function has been derived with the inclusion of the $s$-domain version. Section $\mathrm{V}$ derives analytical formulas for the homogeneous solution to the heat-conduction equation. Section VI presents an $\mathcal{O}(N \lg N)$ algorithm for computing the inhomogeneous solution. Section VII presents an algorithm of the same complexity for computing the homogeneous solution. Also, it introduces the thermal-analysis method LOTAGre. Section VIII experimentally demonstrates the accuracy and speed advantage of LOTAGre, in comparison with a commercial computational fluid dynamics (CFD) tool, called FLUENT, as well as other Green's function-based thermal-analysis methods.

\section{Chip-Level Thermal-Analysis Issue}

This paper mainly considers the steady-state thermalanalysis problem and provides a fast chip-level thermalanalysis method of $\mathcal{O}(N \lg N)$ complexity, where $N$ is the number of blocks that discretize the heat-source and temperature-observation regions. The provided thermalanalysis method can be integrated into inner-loop thermalanalysis procedures in a thermal-aware chip design flow, such as thermal-aware floor planning and cell placement and the analysis of signal-pattern dependent power dissipation [14], [34].

\section{A. Heat-Conduction Equation for Chip-Level Steady-State Thermal Analysis}

In thermal analysis, a given chip can be described by an MTM, which is shown in Fig. 1(b). Fig. 1(a) illustrates two chip examples: Example A illustrates the use of wire-bonding technique; and example B illustrates the use of flip-chip packaging technique [35], [36]. The MTM can describe both types of chips, whereas the STM, i.e., a reduced MTM of one layer, is often used in traditional Green's function-based thermalanalysis methods. The STM can include only the chip active

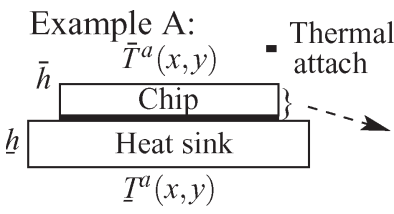

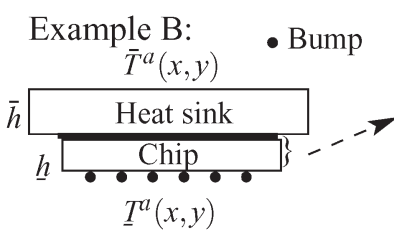

(a)

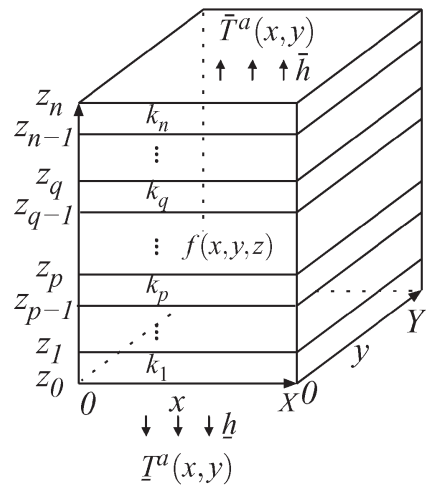

(b)
Fig. 1. Illustration of the MTM: (a) Two chip examples and (b) MTM, with power density distribution given by $f(x, y, z)$ and ambient temperature functions given by $\bar{T}^{a}(x, y)$ and $\underline{T}^{a}(x, y)$.

region into analysis by approximating the thermal effects of the other chip regions and the packaging materials with the heat transfer rates at the chip top and bottom surfaces. In contrast, the MTM can include the entire chip region and the multiplelayer packaging materials. Therefore, this paper uses the MTM shown in Fig. 1(b) to model a given chip.

Given a chip, its steady-state temperature distribution can be obtained by solving the 3-D time-independent heat-conduction equation [17], which in the Cartesian coordinate system is given by

$$
\nabla \cdot[k(z) \nabla T(x, y, z)]=-f(x, y, z)
$$

where $T$ denotes temperature (in kelvin or $\mathrm{K}$ ); the small letter $k$ denotes the material thermal conductivity (in $\mathrm{W} / \mathrm{m} \mathrm{K}$ ); and $f$ denotes the heat-source power density (in watts per meters cubed). In the given chip, thermal conductivity $k$ is a piecewise constant function, with $k$ in each layer assigned a constant value. As shown in Fig. 1(b), $k$ is only $z$-axis dependent, with $k(z)=k_{m}$ for $z_{m-1}<z<z_{m}$ and $1 \leq m \leq n$.

The top ambient temperature $\bar{T}^{a}(x, y)$ and the bottom ambient temperature $T^{a}(x, y)$ have often been specified as the same constant in traditional Green's function-based thermalanalysis methods. In reality, $\bar{T}^{a}$ and $\underline{T}^{a}$ may be considerably different and have large spatial variations in the $x-y$ plane, due to the temperature gradients inside the simulated chip and the inequity of heat flow from different chip surfaces to the outer environment. Therefore, this paper represents $\bar{T}^{a}$ and $\underline{T}^{a}$ by 2-D functions, which have many benefits. For example, the temperature of each bump in example B in Fig. 1(a) can be set as the ambient temperature for the tile where the bump is located. Therefore, the temperatures of all the bumps can be completely incorporated into thermal analysis by a corresponding 2-D function, in contrast with the conventional methods that approximate those different bump temperatures by only the same constant value. This paper reasonably assumes that the four chip sidewalls are surrounded by the same ambient temperature. This chip-sidewall ambient temperature is chosen as the reference temperature, i.e., $T$ in (1), $\bar{T}^{a}$ and $\underline{T}^{a}$ are the temperature deviations from the sidewall ambient temperature. 


\section{B. Heat-Conduction BCs}

Three sets of BCs are specified for the heat conduction equation (1): 1) BCs for the four chip sidewalls, named sidewall $\mathrm{BCs}$; 2) BCs for the horizontal inner interfaces between adjacent layers, named interlayer BCs; and 3) BCs for the chip top and bottom surfaces, named top-bottom BCs. These BCs are introduced in the following.

1) Sidewall BCs: If the four chip sidewalls, i.e., $x=0, X$ and $y=0, Y$, are insulated from the ambient environment, the Neumann's sidewall BCs are specified

$$
\left.\frac{\partial T}{\partial x}\right|_{x=0, X}=0 \text { and }\left.\frac{\partial T}{\partial y}\right|_{y=0, Y}=0 .
$$

If the four sidewalls remain at a specific sidewall ambient temperature, the Dirichlet's sidewall BCs should be specified

$$
\left.T\right|_{x=0, X}=0 \text { and }\left.T\right|_{y=0, Y}=0
$$

where the right-hand sides are zero, because the sidewall ambient temperature has been chosen as the reference value.

In the above, capital letters $X$ and $Y$ denote the dimensions of the chip along the $x$ and $y$ axes, respectively. The chip vertical dimension is specified by interval $\left[z_{0}, z_{n}\right]$. Without explicitly elaborating, the Neumann's sidewall BCs (2) are used in this paper to solve the heat-conduction equation.

2) Interlayer BCs: For any horizontal inner interface $z_{m}$, for $0<m<n$, interlayer BCs are specified to ensure the continuity of temperature, described by (4a), and the continuity of per unit-area heat flux through the interface, described by $(4 b)$ as

$$
\begin{aligned}
\left.T\right|_{z=z_{m+}} & =\left.T\right|_{z=z_{m-}} \\
\left.k_{m+1} \frac{\partial T}{\partial z}\right|_{z=z_{m+}} & =\left.k_{m} \frac{\partial T}{\partial z}\right|_{z=z_{m-}} .
\end{aligned}
$$

3) Top-Bottom BCs: At the chip top and bottom surfaces, the phenomena of heat transfer with the ambient environment are described by heat-convection BCs

$$
\begin{array}{r}
k_{1} \frac{\partial T}{\partial z}-\left.\underline{h} T\right|_{z=z_{0}}=-\underline{h} \underline{T}^{a}(x, y) \\
-k_{n} \frac{\partial T}{\partial z}--\left.\bar{h} T\right|_{z=z_{n}}=-\bar{h} \bar{T}^{a}(x, y) .
\end{array}
$$

Here, $\underline{h}$ (or $\bar{h}$ ) is the heat transfer rate between the chip bottom (or top) surface $z_{0}$ (or $z_{n}$ ) and the ambient, with units watts per squared meters.

Given a power density distribution $f(x, y, z)$, the chip temperature distribution $T(x, y, z)$ can be solved from the heat conduction (1), under the three sets of heat-conduction BCs. Since grid-based thermal-analysis methods normally require volume or surface meshing, employing large amounts of grids in modeling those regions that are of little interest renders the grid-based methods inefficient for performing chip-level thermal analysis. Contrastingly, the application of Green's function in thermal analysis helps discern the important and impertinent chip regions, thereby improving the thermal simulation time by orders of magnitude [14]-[16]. In the following sections, a fast Green's function-based thermal-analysis method will be introduced.

\section{GReEn's Function-BASED Thermal AnAlysis}

Consider the top-bottom BCs in (5). Since they are inhomogeneous, chip temperature distribution is separated into two parts: 1) an inhomogeneous solution, denoted by $T^{\mathrm{i}}$, which satisfies the Poisson's equation imposed with zero top and bottom ambient temperatures and 2) a homogeneous solution, denoted by $T^{\mathrm{h}}$, which satisfies the Laplace's equation imposed with the given 2-D top and bottom ambient temperature functions. Therefore, at a given chip location $(x, y, z)$, there exist an inhomogeneous temperature $T^{\mathrm{i}}(x, y, z)$ and a homogeneous temperature $T^{\mathrm{h}}(x, y, z)$

$$
T(x, y, z)=T^{\mathrm{i}}(x, y, z)+T^{\mathrm{h}}(x, y, z) .
$$

\section{A. Inhomogeneous Solution}

The inhomogeneous solution $T^{\mathrm{i}}$ satisfies the heat conduction equation (1) and all three sets of heat-conduction BCs, but with the right-hand sides of the top-bottom BCs (5) replaced by zeros. The complete equations for $T^{\mathrm{i}}$, except the sidewall and interlayer BCs, are described as

$$
\begin{aligned}
& \nabla \cdot\left[k(z) \nabla T^{\mathrm{i}}(x, y, z)\right]=-f(x, y, z) \\
& k_{1} \frac{\partial T^{\mathrm{i}}}{\partial z}-\left.\underline{h} T^{\mathrm{i}}\right|_{z=z_{0}}=0 \\
&-k_{n} \frac{\partial T^{\mathrm{i}}}{\partial z}-\left.\bar{h} T^{\mathrm{i}}\right|_{z=z_{n}}=0 .
\end{aligned}
$$

The inhomogeneous solution $T^{\mathrm{i}}$ can be obtained by using the Green's function of (6), which is called in this paper as the heat-conduction Green's function, being denoted by $G\left(x, y, z \mid x^{\prime}, y^{\prime}, z^{\prime}\right)$. $G$ corresponds to the temperature distribution of the chip under the top-bottom BCs (6b) and (6c), when the power density distribution $f(x, y, z)$ is a Dirac delta function $\delta\left(x-x^{\prime}, y-y^{\prime}, z-z^{\prime}\right)$, which denotes a unit-strength heat source at chip location $\left(x^{\prime}, y^{\prime}, z^{\prime}\right)$. The complete equations for $G$, except the sidewall $\mathrm{BCs}$, are given in the following:

$$
\begin{aligned}
& \nabla \cdot\left[k(z) \nabla G\left(x, y, z \mid x^{\prime}, y^{\prime}, z^{\prime}\right)\right]=-\delta\left(x-x^{\prime}, y-y^{\prime}, z-z^{\prime}\right) \\
&\left.G\right|_{z=z_{m+}}=\left.G\right|_{z=z_{m-}} \\
&\left.k_{m+1} \frac{\partial G}{\partial z}\right|_{z=z_{m+}}=\left.k_{m} \frac{\partial G}{\partial z}\right|_{z=z_{m-}} \\
& k_{1} \frac{\partial G}{\partial z}-\left.\underline{h} G\right|_{z=z_{0}}=0 \\
&-k_{n} \frac{\partial G}{\partial z}-\left.\bar{h} G\right|_{z=z_{n}}=0
\end{aligned}
$$


Given the heat-conduction Green's function $G$, the inhomogeneous solution $T^{\mathrm{i}}(x, y, z)$ can then be represented as the spatial convolution of power density distribution $f$ with $G$

$T^{\mathrm{i}}(x, y, z)=\int_{\mathcal{V}} G\left(x, y, z \mid x^{\prime}, y^{\prime}, z^{\prime}\right) f\left(x^{\prime}, y^{\prime}, z^{\prime}\right) d x^{\prime} d y^{\prime} d z^{\prime}$

where $\mathcal{V}$ denotes the entire volume space of the simulated chip.

\section{B. Homogeneous Solution}

The homogeneous solution $T^{\mathrm{h}}$ satisfies the homogeneous heat-conduction equation obtained by setting the right-hand side of (1) to zero. Meanwhile, $T^{\mathrm{h}}$ satisfies the three sets of heat-conduction BCs given in Section II-B. In summary, the complete equations governing $T^{\mathrm{h}}$ are described below as

$$
\begin{aligned}
\nabla \cdot\left[k(z) \nabla T^{\mathrm{h}}(x, y, z)\right] & =0 \\
k_{1} \frac{\partial T^{\mathrm{h}}}{\partial z}-\left.\underline{h} T^{\mathrm{h}}\right|_{z=z_{0}} & =-\underline{h} \underline{T}^{a}(x, y) \\
-k_{n} \frac{\partial T^{\mathrm{h}}}{\partial z}-\left.\bar{h} T^{\mathrm{h}}\right|_{z=z_{n}} & =-\bar{h} \bar{T}^{a}(x, y) \\
\left.T^{\mathrm{h}}\right|_{z=z_{m+}} & =\left.T^{\mathrm{h}}\right|_{z=z_{m-}} \\
\left.k_{m+1} \frac{\partial T^{\mathrm{h}}}{\partial z}\right|_{z=z_{m+}} & =\left.k_{m} \frac{\partial T^{\mathrm{h}}}{\partial z}\right|_{z=z_{m-}} \\
\left.\frac{\partial T^{\mathrm{h}}}{\partial x}\right|_{x=0, X} & =0 \\
\left.\frac{\partial T^{\mathrm{h}}}{\partial y}\right|_{y=0, Y} & =0 .
\end{aligned}
$$

Owing to the principle of superposition, separating the entire temperature solution $T$ into two parts has allowed representing the inhomogeneous solution $T^{\mathrm{i}}$ by the convolution integral (8) and representing $T$ by the summation of $T^{\mathrm{i}}$ and $T^{\mathrm{h}}$.

\section{Discussion on Green's Function-Based Thermal Analysis}

By assuming a zero homogeneous solution $T^{\mathrm{h}}$, traditional Green's function-based thermal-analysis methods mainly consider the inhomogeneous solution $T^{\mathrm{i}}$ [14]-[16]. This paper additionally addresses the homogeneous solution $T^{\mathrm{h}}$ and uses general 2-D functions to describe the ambient temperatures at the top and bottom surfaces of the simulated chip. Since the homogeneous solution $T^{\mathrm{h}}$ and the inhomogeneous solution $T^{\mathrm{i}}$ are independent, and the former does not depend on the power density distribution $f, T^{\mathrm{h}}$ should be computed only once for a given ambient condition.

The Green's function for the Poisson's equation has been discussed in the literature. In [37], the Green's function for the 2-D Poisson's equation was considered, under various types of BCs and geometrical configurations. In [24], the Green's function for the 3-D Poisson's equation was derived for parasitic extraction, under the assumption that the chip horizontal dimensions were infinite. For substrate modeling, in [25],
Green's function was derived with the Neumann's sidewall BCs imposed and with the potential or potential gradient at the chip top and bottom surfaces set to zero; and in [26], numerical stability issue was further considered. In thermal analysis, however, the two surfaces of the chip should be imposed with general heat-convection BCs. Considering one homogeneous heat-conduction material, [14] derived the Green's function by assuming a heat-insulation $\mathrm{BC}$ on the chip top surface, and [16] derived the Green's function under the Neumann's sidewall BCs. To consider heterogeneous materials, the multilayer heatconduction Green's function will be derived in this paper.

In the following sections, this paper at first derives a set of analytical formulas for the inhomogeneous solution $T^{\mathrm{i}}$ and then for the homogeneous solution $T^{\mathrm{h}}$. For the derivation of $T^{\mathrm{i}}$, this paper mainly considers the required multilayer heat-conduction Green's function, including the $s$-domain version.

\section{Deriving The Inhomogeneous Solution}

The inhomogeneous solution $T^{\mathrm{i}}$ has been given in the form of (8). To solve the required heat-conduction Green's function $G$ from its governing equations in (7), this paper employs the eigen-expansion technique [38]. Consider that the heat conduction equation (1) is imposed with the Neumann's sidewall BCs (2), which approximate that there is no heat transfer between the simulated chip and the surrounding environment via the four chip sidewalls, because the chip thickness is way smaller than its horizontal dimensions. Then, $G$ should satisfy the following sidewall BCs:

$$
\begin{aligned}
& \left.\frac{\partial G\left(x, y, z \mid x^{\prime}, y^{\prime}, z^{\prime}\right)}{\partial x}\right|_{x=0, X}=0 \\
& \left.\frac{\partial G\left(x, y, z \mid x^{\prime}, y^{\prime}, z^{\prime}\right)}{\partial y}\right|_{y=0, Y}=0 .
\end{aligned}
$$

Consequentially, orthogonal cosine functions are chosen as eigenfunctions.

\section{A. Eigen-Expansion of Heat-Conduction Green's Function}

The domain of the heat-conduction Green's function $G\left(x, y, z \mid x^{\prime}, y^{\prime}, z^{\prime}\right)$ was initially limited to the entire chip volume space $\mathcal{V}$. To obtain an eigen-expansion of $G$, the domain of $G$ is expanded from space $\mathcal{V}$ to the entire 3-D space, and $G$ is expanded to a periodic even function of $x$ of period $2 X$, as well as a periodic even function of $y$ of period $2 Y$. Then, the following eigen-expansion of $G$ results:

$$
G\left(x, y, z \mid x^{\prime}, y^{\prime}, z^{\prime}\right)=\sum_{i=0}^{\infty} \sum_{j=0}^{\infty} \phi_{i j}(x, y) G_{i j}\left(z \mid x^{\prime}, y^{\prime}, z^{\prime}\right) .
$$

Here, $G_{i j}$ is the eigen-expansion coefficient and $\phi_{i j}$ is the eigenfunction defined by $\phi_{i j}(x, y)=$ $\cos (i \pi x / X) \cos (j \pi y / Y)$. Eigen-expansion (11) ensures that $G$ satisfies the Neumann's sidewall BCs in (10).

Insert (11) into (7). Then, multiplying the two sides of (7a) by $\phi_{i j}(x, y)$ and integrating over the $x$ and $y$ dimensions of the chip leads to (12a), due to the orthogonality of eigenfunctions 


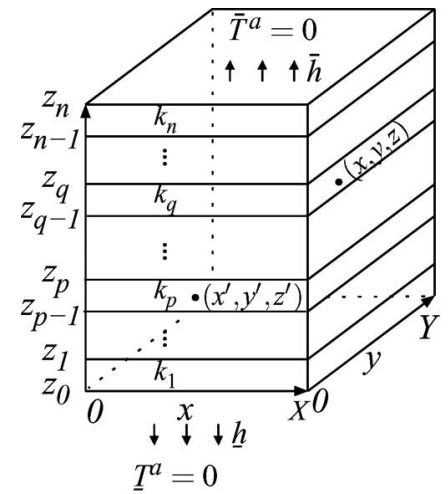

(a)

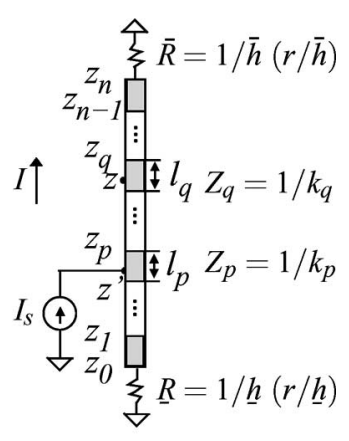

(b)
Fig. 2. Equivalent circuit for deriving the eigen-expansion coefficient $G_{i j}$ : (a) MTM for the 3-D governing equations in (7), with a Dirac delta heat source imposed at the location $\left(x^{\prime}, y^{\prime}, z^{\prime}\right)$ and the ambient temperatures set to zero. (b) Equivalent circuit that describes the 1-D governing equations in (12) (small letter $z$ denotes a location, while capital letter $Z$ denotes TL characteristic impedance).

[38]. After simplifying the remaining equations in (7), the complete equations governing the eigen-expansion coefficient $G_{i j}$ are obtained

$$
\begin{aligned}
\frac{\partial^{2} G_{i j}\left(z \mid x^{\prime}, y^{\prime}, z^{\prime}\right)}{\partial z^{2}} & \\
-\gamma_{i j}^{2} G_{i j}\left(z \mid x^{\prime}, y^{\prime}, z^{\prime}\right) & =-\frac{c_{i j} \phi_{i j}\left(x^{\prime}, y^{\prime}\right) \delta\left(z-z^{\prime}\right)}{k(z)} \\
\left.G_{i j}\right|_{z=z_{m+}} & =\left.G_{i j}\right|_{z=z_{m-}} \\
\left.k_{m+1} \frac{d G_{i j}}{d z}\right|_{z=z_{m+}} & =\left.k_{m} \frac{d G_{i j}}{d z}\right|_{z=z_{m-}} \\
k_{1} \frac{\partial G_{i j}}{d z}-\left.\underline{h} G_{i j}\right|_{z=z_{0}} & =0 \\
-k_{n} \frac{\partial G_{i j}}{d z}-\left.\bar{h} G_{i j}\right|_{z=z_{n}} & =0 .
\end{aligned}
$$

Here, $\gamma_{i j}=\sqrt{\left(i^{2} \pi^{2} / X^{2}\right)+\left(j^{2} \pi^{2} / Y^{2}\right)}$ and $c_{i j}=2^{2-\delta_{i 0}-\delta_{j 0}} /$ $X Y$, where $\delta_{i 0}$ and $\delta_{j 0}$ are Kronecker deltas. After the eigenexpansion coefficient $G_{i j}$ is solved from (12), $G$ can be obtained from (11).

The eigen-expansion coefficient $G_{i j}$ is derived in the following.

\section{B. Eigen-Expansion Coefficient $G_{i j}(i=j=0)$}

When $i=j=0, \gamma_{i j}$ becomes zero. Then, the governing equations in (12) can be shown to be equivalent to the circuit equations for an $n$-section nonuniform line conductor of per unit-length (PUL) conductance $k(z)$. Each section of the line corresponds to one layer in the MTM in Fig. 2(a), which is for the 3-D governing equations in (7), and has a length equal to that layer's thickness. The two ends of the line are terminated by two resistors of resistances $\underline{R}=1 / \underline{h}$ and $\bar{R}=1 / \bar{h}$. Assume the source location $\left(x^{\prime}, y^{\prime}, z^{\prime}\right)$ is in the $p$ th layer of the MTM and the target location $(x, y, z)$ is in the $q$ th layer of the MTM. Then, at the location $z^{\prime}$ in the $p$ th section of the line conductor, there is a current source input $I_{s}$ of intensity $c_{00} \phi_{00}\left(x^{\prime}, y^{\prime}\right)$. Fig. 2(b) shows the equivalent line conductor circuit. The voltage and current at the location $z$ of the shown line conductor, $V(z)$ and $I(z)$, satisfy

$$
\begin{aligned}
& \frac{\partial V(z)}{\partial z}=-\frac{I(z)}{k(z)} \\
& \frac{\partial I(z)}{\partial z}=I_{s} \delta\left(z-z^{\prime}\right) .
\end{aligned}
$$

Therefore, the eigen-expansion coefficient $G_{i j}\left(z \mid x^{\prime}, y^{\prime}, z\right)$ corresponds to the voltage at the location $z$ in the $q$ th section of the line conductor; (12a) corresponds to the Kirchhoff's current law at that location; (12b) and (12c) are equivalent to the current and voltage continuity conditions at the interface between the $m$ th and the $(m+1)$ th line sections. The last two equations in (12) correspond to the circuit equations governing the two ends of the line.

The eigen-expansion coefficient $G_{i j}\left(z \mid x^{\prime}, y^{\prime}, z^{\prime}\right)$ is consequentially obtained by solving the voltage at the location $z$ in the line conductor. The formula for $G_{i j}$ is given by

$$
G_{00}\left(z \mid x^{\prime}, y^{\prime}, z^{\prime}\right)=c_{00} \phi_{00}\left(x^{\prime}, y^{\prime}\right) H_{00}\left(z \mid z^{\prime}\right)
$$

where $H_{00}$ is the transfer impedance of the line conductor from the location $z^{\prime}$ to the location $z$ given by

$$
H_{00}\left(z \mid z^{\prime}\right)=\frac{\left[\underline{Z}_{p}+Z_{p}\left(z^{\prime}-z_{p-1}\right)\right]\left[\bar{Z}_{q}+Z_{q}\left(z_{q}-z\right)\right]}{\underline{Z}_{p}+Z_{p} l_{p}+\bar{Z}_{q}} .
$$

The symbols used previously are explained hereafter. In this paper, when a nonuniform line (either a line conductor or a transmission line) is used in an equivalent circuit, for the $m$ th line section, $\underline{Z}_{m}$ denotes the input impedance seen from that section's bottom boundary toward the bottom side of the equivalent circuit; $\bar{Z}_{m}$ denotes the input impedance seen from that section's top boundary toward the top side of the circuit; $Z_{m}$ denotes the characteristic impedance of that section; and $l_{m}$ is the section length. There are two special cases: $\bar{Z}_{0}$ denotes the input impedance seen from the location $z=z_{0}$ to the top side of the circuit, and $\underline{Z}_{n+1}$ denotes the input impedance seen from the location $z=z_{n}$ to the bottom side of the circuit. For the equivalent line conductor circuit shown in Fig. 2(b), $\bar{Z}_{q}=\bar{R}+\sum_{m=q+1}^{n} Z_{m}$ and $\underline{Z}_{q}=\underline{R}+\sum_{m=1}^{q-1} Z_{m}$.

\section{Eigen-Expansion Coefficient $G_{i j}(i+j>0)$}

When $i+j>0$, a similar circuit equivalence that will facilitate solving $G_{i j}$ from (12) can be established from the similarity between (12) and the following transmission-line (TL) equations:

$$
\begin{aligned}
& \frac{\partial V(z)}{\partial z}=-(R+s L) I(z) \\
& \frac{\partial I(z)}{\partial z}=-(G+s C) V(z)+I_{s} \delta\left(z-z^{\prime}\right)
\end{aligned}
$$


or in the form of $\left(\partial^{2} V(z) / \partial z^{2}\right)-\gamma^{2} V(z)=-\gamma Z I_{s} \delta\left(z-z^{\prime}\right)$, where the propagation constant and the characteristic impedance of the TL are given by $\gamma=\sqrt{(R+s L)(G+s C)}$ and $Z=\sqrt{(R+S L) /(G+s C)}$, respectively [39], [40]. The governing equations of $G_{i j}$ in (12) can be shown to be equivalent to the circuit equations for an $n$-section nonuniform TL. In the equivalent TL circuit, each line section corresponds to one layer in the MTM in Fig. 2(a) and has a length equal to that layer's thickness. The PUL parameters of the $m$ th TL section, $R, L, C$, and $G$ satisfy $\sqrt{R G}=\gamma_{i j}, \sqrt{R / G}=Z_{m}=1 / k_{m}$, and $L=C=0$. The two ends of the TL are terminated by two resistors of resistances $\bar{R}=\gamma / \bar{h}$ and $\underline{R}=\gamma / \underline{h}$, and at the location $z^{\prime}$ in the $p$ th section of the TL, a current source input of intensity $I_{s}=\left(c_{i j} / \gamma_{i j}\right) \phi_{i j}\left(x^{\prime}, y^{\prime}\right)$ is imposed. Consequentially, this paper uses the same circuit diagram that is for the line conductor circuit to illustrate the equivalent TL circuit, as again shown by Fig. 2(b). Note that the two terminating resistors choose the resistance values enclosed in the parentheses.

According to Fig. 2(b), the eigen-expansion coefficient $G_{i j}\left(z \mid x^{\prime}, y^{\prime}, z^{\prime}\right)$ corresponds to the voltage at the location $z$ of the TL under a current source input $I_{s}$ at the location $z^{\prime}$. Therefore, with the help of the transfer impedance of the TL, $G_{i j}\left(z \mid x^{\prime}, y^{\prime}, z^{\prime}\right)$, for $i+j>0$, is obtained in the following:

$$
G_{i j}\left(z \mid x^{\prime}, y^{\prime}, z^{\prime}\right)=c_{i j} \phi_{i j}\left(x^{\prime}, y^{\prime}\right) H_{i j}\left(z \mid z^{\prime}\right)
$$

where $H_{i j}$ is the normalized transfer impedance from the location $z^{\prime}$ to the location $z$ of the TL by the propagation constant $\gamma$, with $\gamma=\gamma_{i j}=\sqrt{\left(i^{2} \pi^{2} / X^{2}\right)+\left(j^{2} \pi^{2} / Y^{2}\right)}$. The normalized transfer impedance $H_{i j}$ is given by

$H_{i j}\left(z \mid z^{\prime}\right)=\xi \frac{\left(\underline{Z}_{p} \cosh \gamma \underline{l}+Z_{p} \sinh \gamma \underline{l}\right)\left(\bar{Z}_{q} \cosh \gamma \bar{l}+Z_{q} \sinh \gamma \bar{l}\right)}{Z_{p}\left(\underline{Z}_{p}+\bar{Z}_{p}\right) \cosh \gamma l_{p}+\left(Z_{p}^{2}+\underline{Z}_{p} \bar{Z}_{p}\right) \sinh \gamma l_{p}}$

where $\underline{l}=z^{\prime}-z_{p-1}, \bar{l}=z_{q}-z$, and $\xi$ is given by

$$
\xi=\frac{Z_{p} \prod_{m=p}^{q-1} \bar{Z}_{m}}{\gamma \prod_{m=p+1}^{q}\left(\bar{Z}_{m} \cosh \gamma l_{m}+Z_{m} \sinh \gamma l_{m}\right)} .
$$

Those input impedances $\underline{Z}$ s and $\bar{Z}$ s have recurrence formulas: For one TL section in a nonuniform TL, the input impedance at its one boundary, denoted by $Z_{\text {in }}$, has a recurrence formula

$$
Z_{\text {in }}=\mathrm{ZC} \frac{\mathrm{ZL}+\mathrm{ZC} \tanh \gamma L}{\mathrm{ZC}+\mathrm{ZL} \tanh \gamma L}
$$

where $\mathrm{ZL}$ is the load impedance at the other boundary, $\mathrm{ZC}$ is the characteristic impedance of this TL section, and $L$ is the section length.

To demonstrate the multilayer heat-conduction Green's function derived here, the following uses a multilayer structure considered by Albers as an example [28].

\section{Surface-Temperature Solution for a Multilayer Structure by Employing the Multilayer Heat-Conduction Green's Function}

In [28], Albers gave a recursion relation for the steady-state surface temperature of a multilayer structure and showed that the recursion analytically agrees with Kokkas' solution for up to three layers [27]. By using the multilayer Green's function derived previously, the following obtains a surface-temperature solution that agrees with Albers' recursion relation for arbitrary number of layers.

The multilayer structure used by Albers is a special case of the MTM in Fig. 1(b) by letting $\bar{h}=0, \underline{h}=\infty, \bar{T}^{a}(x, y)=$ $\underline{T}^{a}(x, y)=0$, and $f(x, y, z)=P_{0} u(x, y) \delta\left(z-z_{n}\right)$. Therefore, according to (8), (11), (13), and (15), the surface temperature can be given by

$T\left(x, y, z_{n}\right)=P_{0} \sum_{i=0}^{\infty} \sum_{j=0}^{\infty} c_{i j} U(i, j) \phi_{i j}(x, y) H_{i j}\left(z_{n} \mid z_{n}\right)$

where $U(i, j)=\int_{0}^{X} \int_{0}^{Y} u\left(x^{\prime}, y^{\prime}\right) \phi_{i j}\left(x^{\prime}, y^{\prime}\right) d x^{\prime} d y^{\prime}$.

Inserting $\bar{Z}_{n}=\infty$ into (14), $H_{00}\left(z_{n} \mid z_{n}\right)$ is obtained as

$$
H_{00}\left(z_{n} \mid z_{n}\right)=\underline{Z}_{n}+Z_{n} l_{n}=\sum_{m=1}^{n} \frac{l_{m}}{k_{m}} .
$$

Inserting $\underline{l}=l_{n}$ and $\bar{Z}_{n}=\infty$ into (16) and employing (18), $H_{i j}\left(z_{n} \mid z_{n}\right)$, for $i+j>0$, is obtained as

$$
H_{i j}\left(z_{n} \mid z_{n}\right)=\frac{1}{\gamma} \underline{Z}_{n+1}=\frac{1}{\gamma} Z_{n} \frac{\underline{Z}_{n}+Z_{n} \tanh \gamma l_{n}}{Z_{n}+\underline{Z}_{n} \tanh \gamma l_{n}} .
$$

Note that since $\underline{h}=\infty, \underline{Z}_{2}=Z_{1} \tanh \gamma l_{1}$. Clearly, after a simple transformation $\tau_{m}=\underline{Z}_{m+1} k_{m}$, (19), (20), and (21), obtained by employing the multilayer Green's function derived in this paper, are the same as the analytical formulas derived by Albers [28]. In terms of numerically computing the surface temperature, Kokkas presented extensive numerical results in [27] and discussed the convergence issue of the series expansion (19).

\section{E. Discussion on Deriving the Multilayer Heat-Conduction Green's Function}

By integrating the eigen-expansion technique and classic TL theory, this paper has derived the multilayer heat-conduction Green's function, with the Neumann's sidewall BCs (2) being imposed. By using the multilayer Green's function, the steadystate temperature distribution due to an arbitrary power density distribution can be obtained. For example, this paper has derived a surface-temperature solution that agrees with Albers' recursion relation for a multilayer structure. The methodology of deriving the multilayer Green's function can still be applied, when other types of sidewall BCs are considered. For example, consider using the Dirichlet's sidewall BCs (3), i.e., assuming that the sidewall temperatures remain a constant value. After the eigenfunction is redefined as $\phi_{i j}(x, y)=$ $\sin (i \pi x / X) \sin (j \pi y / Y)$, the same form of equations as those in (12) still results in governing $G_{i j}$. Therefore, the heatconduction Green's function under the Dirichlet's sidewall BCs can be derived in the same way as described previously. Szekely et al. [41] have presented a comprehensive library of eigenfunctions, which can be employed for deriving Green's function under other sidewall BCs. 
In calculating the Green's function derived here, the relationship between the eigen-expansion coefficients and circuit transfer functions leads to the following observation: When heat transfer rate $\underline{h}$ or $\bar{h}$ is zero or close to zero, the load impedances at the end sides of the equivalent circuits become infinite or too large to be represented in a floating-point number system; therefore, instead of using impedance formulations, the use of admittance functions in calculation can avoid numerical overflows. The following asymptotic estimation about $H_{i j}$ can also be made by expanding the hyperbolic functions in the explicit formulas (16) and (17): as $\gamma$ increases, $\bar{Z} \mathrm{~s}$ and $\underline{Z} \mathrm{~s}$ are close to some constant values according to (18); therefore, when $z \neq z^{\prime}$, the trend of $H_{i j}$ is dominated by the exponential decrease at a rate of $\mathrm{e}^{\gamma\left(\underline{l}+\bar{l}-\sum_{m=p}^{q} l_{m}\right)}$, and when $z=z^{\prime}$, the decrease of $H_{i j}$ is due to the $1 / \gamma$ term in (17). In the special case that $z=z^{\prime}=z_{n}, H_{i j}$ has a concise form (21), by which $H_{i j}$ can be efficiently computed.

Proceeding as described, the multilayer Green's function for the time-dependent heat-conduction equation is derived as follows.

\section{F. Derivation of the s-Domain Multilayer Heat-Conduction Green's Function}

The time-dependent heat-conduction equation for the MTM in Fig. 1(b) is described by

$$
\nabla \cdot[k(z) \nabla T(x, y, z, t)]-\rho(z) c(z) \frac{\partial T(x, y, z, t)}{\partial t}=-f(x, y, z, t)
$$

where $\rho$ is the density of the material and $c$ is the specific heat. The multilayer Green's function for (22), denoted by $\mathcal{G}\left(x, y, z, t \mid x^{\prime}, y^{\prime}, z^{\prime}\right)$, is the temperature solution to (22) under zero initial temperature distribution and zero ambient temperatures, when a Dirac delta source is imposed as the power density distribution, i.e., $f(x, y, z, t)=\delta\left(x-x^{\prime}, y-y^{\prime}, z-z^{\prime}, t\right)$.

Consequentially, in the $s$-domain, the Laplace transform of $\mathcal{G}\left(x, y, z, t \mid x^{\prime}, y^{\prime}, z^{\prime}\right)$, or the $s$-domain Green's function $\mathcal{G}\left(x, y, z, s \mid x^{\prime}, y^{\prime}, z^{\prime}\right)$, satisfies

$$
\nabla^{2} \mathcal{G}-\frac{s}{d(z)} \mathcal{G}=\frac{\delta\left(x-x^{\prime}, y-y^{\prime}, z-z^{\prime}\right)}{k(z)}
$$

where $d$ is the material thermal diffusivity, with $d(z)=$ $k(z) /(\rho(z) c(z))$. Insert the eigen-expansion of $\mathcal{G}$

$$
\mathcal{G}\left(x, y, z, s \mid x^{\prime}, y^{\prime}, z^{\prime}\right)=\sum_{i=0}^{\infty} \sum_{j=0}^{\infty} \phi_{i j}(x, y) \mathcal{G}_{i j}\left(z, s \mid x^{\prime}, y^{\prime}, z^{\prime}\right)
$$

into (23). The same set of governing equations as in (12) is obtained for $\mathcal{G}_{i j}$ after defining $\gamma_{i j}=$ $\sqrt{\left(i^{2} \pi^{2} / X^{2}\right)+\left(j^{2} \pi^{2} / Y^{2}\right)+(s / d(z))}$. Therefore, the equivalent circuit in Fig. 2(b) is employed to derive the eigenexpansion coefficient $\mathcal{G}_{i j}$.

Note that since $\gamma_{i j}$ is now $z$-axis dependent, circuit parameters in Fig. 2(b) should be altered in the following way. For the $m$ th line section, its propagation constant and characteristic impedance are specified as $\gamma^{(m)}=\sqrt{\left(i^{2} \pi^{2} / X^{2}\right)+\left(j^{2} \pi^{2} / Y^{2}\right)+\left(s / d_{m}\right)}$ and $Z_{m}=$ $1 /\left(k_{m} \gamma^{(m)}\right)$, respectively. Here, $d_{m}$ is the thermal diffusivity of the material in the $m$ th layer. The intensity of the current source input and the resistances of the two terminating resistors are specified by $I_{s}=c_{i j} \phi_{i j}\left(x^{\prime}, y^{\prime}\right), \bar{R}=1 / \bar{h}$, and $\underline{R}=1 / \underline{h}$, respectively.

The formulas for $\mathcal{G}_{i j}$ obtained here are similar to (15) and (16)

$$
\begin{aligned}
& \mathcal{G}_{i j}\left(z, s \mid x^{\prime}, y^{\prime}, z^{\prime}\right) \\
& \quad=c_{i j} \phi_{i j}\left(x^{\prime}, y^{\prime}\right) \mathcal{H}_{i j}\left(z, s \mid z^{\prime}\right) \\
& \mathcal{H}_{i j}\left(z, s \mid z^{\prime}\right) \\
& =\frac{\eta\left(\underline{Z}_{p} \cosh \gamma^{(p)} \underline{l}+Z_{p} \sinh \gamma^{(p)} \underline{l}\right)}{\left(\underline{Z}_{p}+\bar{Z}_{p}\right) \cosh \gamma^{(p)} l_{p}+\left(Z_{p}+\underline{Z}_{p} \bar{Z}_{p} / Z_{p}\right) \sinh \gamma^{(p)} l_{p}} \\
& \eta=\frac{\left(\bar{Z}_{q} \cosh \gamma^{(q)} \bar{l}+Z_{q} \sinh \gamma^{(q)} \bar{l}\right) \prod_{m=p}^{q-1} \bar{Z}_{m}}{\prod_{m=p+1}^{q}\left(\bar{Z}_{m} \cosh \gamma^{(m)} l_{m}+Z_{m} \sinh \gamma^{(m)} l_{m}\right)}
\end{aligned}
$$

When $\gamma^{(m)}$ goes to zero,(25) can be reformulated by employing the following:

$$
\lim _{\gamma^{(m)} \rightarrow 0} Z_{m} \sinh \gamma^{(m)} l=\frac{l}{k_{m}} .
$$

Apparently, changing the eigenfunction $\phi_{i j}$ will lead to the $s$-domain multilayer Green's function under other types of BCs.

\section{G. Application of the s-Domain Multilayer Green's Function in Computing the Thermal Transfer Impedance}

With the $s$-domain multilayer Green's function, the thermal transfer impedance from an arbitrary-shape input volume iv to an arbitrary-shape output volume ov, denoted by $R_{\mathrm{th}}^{\text {(iv,ov) }}(s)$ in this paper, can be given by

$$
R_{\mathrm{th}}^{(\mathrm{iv}, \mathrm{ov})}(s)=\frac{\int_{\mathrm{ov}} \int_{\mathrm{iv}} \mathcal{G}\left(x, y, z, s \mid x^{\prime}, y^{\prime}, z^{\prime}\right) d x^{\prime} d y^{\prime} d z^{\prime} d x d y d z}{\int_{\mathrm{ov}} d x d y d z \int_{\mathrm{iv}} d x^{\prime} d y^{\prime} d z^{\prime}} .
$$

For the thermal transfer impedance at the top surface of a multilayer structure, Kokkas gave the solution for up to three layers [27], and the general solution for arbitrary number of layers was given implicitly in [29] by a system of linear equations and explicitly in [19] by the products of $2 \times 2$ transfer matrices. Based on (26), the thermal transfer impedance at the surface is obtained below in a concise form.

The multilayer structure used in the literature is a special case of the MTM in Fig. 1(b) by letting $\bar{h}=0$ and $\underline{h}=\infty$. Therefore, at the top surface of the MTM in Fig. 1(b), the thermal transfer impedance to any point pt of location $\left(x, y, z_{n}\right)$ from an arbitrary-shape heat-source region hs of area $\mathcal{A}, R_{\mathrm{th}}^{(\mathrm{hs}, \mathrm{pt})}$, can be obtained by inserting (24) and (25) into (26)

$$
R_{\mathrm{th}}^{(\mathrm{hs}, \mathrm{pt})}(s)=\frac{1}{\mathcal{A}} \sum_{i=0}^{\infty} \sum_{j=0}^{\infty} I_{i j} c_{i j} \phi_{i j}(x, y) \mathcal{H}_{i j}\left(z_{n}, s \mid z_{n}\right)
$$

where $I_{i j}=\int_{\mathrm{hs}} \phi_{i j}\left(x^{\prime}, y^{\prime}\right) d x^{\prime} d y^{\prime}$. 


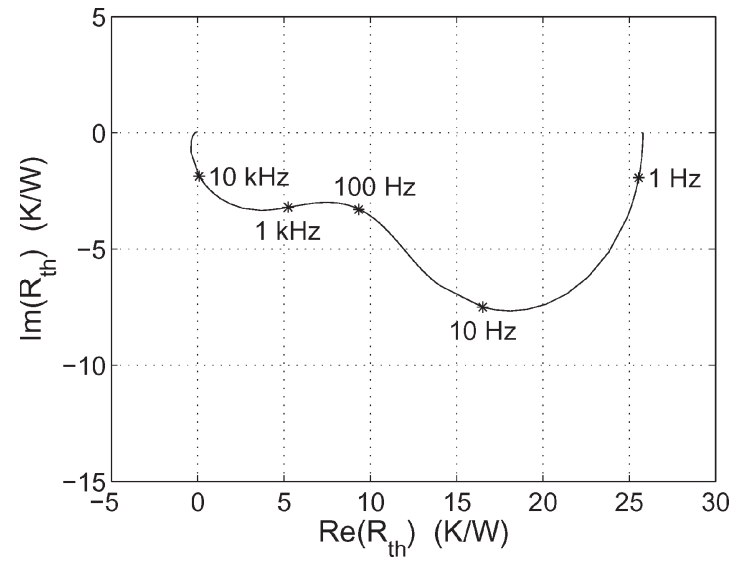

Fig. 3. Complex locus of the thermal impedance for the structure examined in [19], [42], and [43], computed by (27) and (28).

Inserting $\underline{l}=l_{n}, \bar{l}=0$, and $\bar{Z}_{n}=\infty$ into (25) and employing (18), the following formula is obtained:

$$
\mathcal{H}_{i j}\left(z_{n}, s \mid z_{n}\right)=\underline{Z}_{n+1}=Z_{n} \frac{\underline{Z}_{n}+Z_{n} \tanh \gamma^{(n)} l_{n}}{Z_{n}+\underline{Z}_{n} \tanh \gamma^{(n)} l_{n}}
$$

That $\underline{h}=\infty$ leads to $\underline{R}=0$ and $\underline{Z}_{2}=Z_{1} \tanh \gamma^{(1)} l_{1}$. By employing (27) and (28), the thermal transfer impedance at the surface can be efficiently computed. The complex locus results computed by (27) and (28) for an examined structure in the literature [19, Fig. 8], [42, Figs. 5 and 6], [43, Fig. 17] are plotted in Fig. 3, which show good agreement with the results in [19], [42], and [43].

To establish compact thermal models [19], [20] [29], the required thermal-transfer-impedance matrices can also be generated by the $s$-domain multilayer Green's function-based method, formulated in (26). In the same way as the $2 \times 2$ transfer matrix approach in [19], the presented method by (26) leads to fully analytical double Fourier series such as (27). With the explicit formulas derived for the coefficient $\mathcal{H}_{i j}$, the efficiency of establishing compact thermal models can be improved. For example, to compute the surface thermal transfer impedance, a system of equations should be solved in an implicit method, and several times more complex number calculations are required by a transfer matrix method than by (28).

This section has derived the multilayer heat-conduction Green's function with the inclusion of the $s$-domain version and demonstrated the Green's function usage by the known examples in the literature. Since this paper mainly considers the steady-state thermal issue, the Green's function $G\left(x, y, z \mid x^{\prime}, y^{\prime}, z^{\prime}\right)$ derived here will be used in the following sections.

\section{Deriving the Homogeneous Solution}

This section derives the homogeneous solution $T^{\mathrm{h}}$ from (9) by following the same procedure that derives the heatconduction Green's function $G$, i.e., by employing the eigenexpansion technique and TL theory.

\section{A. Eigen-Expansion of the Homogeneous Solution}

The even periodic expansion is similarly applied to the homogeneous solution $T^{\mathrm{h}}(x, y, z)$, and the 2-D top and bottom ambient temperature functions $\bar{T}^{a}(x, y)$ and $\underline{T}^{a}(x, y)$. Then, the eigen-expansions of $T^{\mathrm{h}}, \bar{T}^{a}$, and $\underline{T}^{a}$ are obtained as follows:

$$
\begin{gathered}
T^{\mathrm{h}}(x, y, z)=\sum_{i=0}^{\infty} \sum_{j=0}^{\infty} \phi_{i j}(x, y) t_{i j}^{\mathrm{h}}(z) \\
\bar{T}^{a}(x, y)=\sum_{i=0}^{\infty} \sum_{j=0}^{\infty} \phi_{i j}(x, y) \bar{t}_{i j}^{a} \\
\underline{T}^{a}(x, y)=\sum_{i=0}^{\infty} \sum_{j=0}^{\infty} \phi_{i j}(x, y) \underline{t}_{i j}^{a}
\end{gathered}
$$

where $t_{i j}^{\mathrm{h}}, \bar{t}_{i j}^{a}$, and $\underline{t}_{i j}^{a}$ are eigen-expansion coefficients, and the eigenfunction remains as $\phi_{i j}(x, y)=\cos (i \pi x / X)$. $\cos (j \pi y / Y)$. By the eigen-expansion (29a), $T^{\mathrm{h}}$ automatically satisfies the Neumann's sidewall BCs (9f).

Insert (29) into (9) and eliminate the $\phi_{i j}(x, y)$ terms in those equations. Then, the complete equations governing the eigenexpansion coefficient $t_{i j}^{\mathrm{h}}$ are obtained

$$
\begin{aligned}
\frac{d^{2} t_{i j}^{\mathrm{h}}(z)}{d z^{2}}-\gamma_{i j}^{2} t_{i j}^{\mathrm{h}}(z) & =0 \\
\left.t_{i j}^{\mathrm{h}}\right|_{z=z_{m+}} & =\left.t_{i j}^{\mathrm{h}}\right|_{z=z_{m-}} \\
\left.k_{m+1} \frac{d t_{i j}^{\mathrm{h}}}{d z}\right|_{z=z_{m+}} & =\left.k_{m} \frac{d t_{i j}^{\mathrm{h}}}{d z}\right|_{z=z_{m-}} \\
k_{1} \frac{d t_{i j}^{\mathrm{h}}}{d z}-\left.\underline{h} t_{i j}^{\mathrm{h}}\right|_{z=z_{0}} & =-\underline{h} \underline{t}_{i j}^{a} \\
-k_{n} \frac{d t_{i j}^{\mathrm{h}}(z)}{d z}-\left.\bar{h} t_{i j}^{\mathrm{h}}\right|_{z=z_{n}} & =-\bar{h} \bar{t}_{i j}^{a} .
\end{aligned}
$$

Here, $\gamma_{i j}$ remains as $\gamma_{i j}=\sqrt{\left(i^{2} \pi^{2} / X^{2}\right)+\left(j^{2} \pi^{2} / Y^{2}\right)}$. The homogeneous solution $T^{\mathrm{h}}$ can accordingly be obtained in the following framework: Given the 2-D top and bottom ambient temperature functions $\bar{T}^{a}(x, y)$ and $\underline{T}^{a}(x, y)$, first compute the eigen-expansion coefficients $\bar{t}_{i j}^{a}$ and $\underline{t}_{i j}^{a}$ based on formulas (29b) and (29c); then solve the eigen-expansion coefficient $t_{i j}^{\mathrm{h}}$ from (30). Finally, calculate $T^{\mathrm{h}}(x, y, z)$ according to (29a).

Since $t_{i j}^{\mathrm{h}}$, s governing equations in (30) bear some similarities with (12), $t_{i j}^{\mathrm{h}}$ is solved from (30) by the same means of employing the TL theory.

\section{B. Eigen-Expansion Coefficient $t_{i j}^{\mathrm{h}}(i=j=0)$}

Let $i=j=0$, and $\gamma_{i j}$ becomes zero. Then, the governing equations in (30) can be shown to be equivalent to the circuit equations for an $n$-section nonuniform line conductor of PUL 


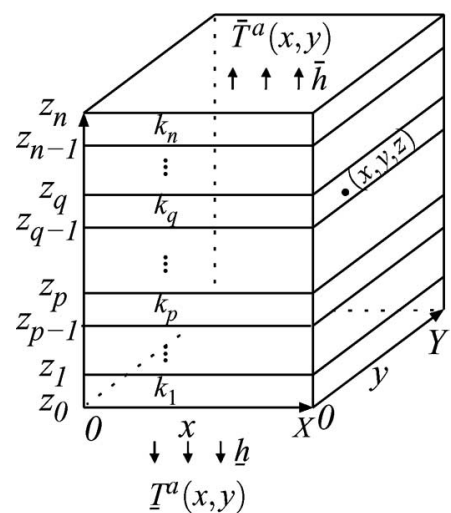

(a)

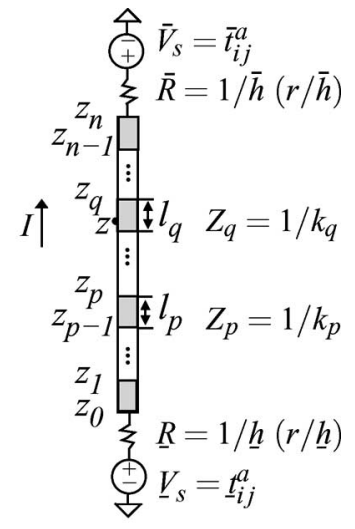

(b)
Fig. 4. Equivalent circuit for deriving the eigen-expansion coefficient $t_{i j}^{\mathrm{h}}$ : (a) MTM for the 3-D governing equations in (9), with the top and bottom ambient temperature functions imposed and the power density distribution set to zero. (b) Equivalent circuit that describes the 1-D governing equations in (30).

conductance $k(z)$. The two ends of the line conductor connect to two resistors of resistances $\bar{R}=1 / \bar{h}$ and $\underline{R}=1 / \underline{h}$, and the two resistors are driven by two voltage sources of magnitudes $\bar{V}_{s}=\bar{t}_{i j}^{a}$ and $\underline{V}_{s}=\underline{t}_{i j}^{a}$. Fig. 4 shows the equivalent line conductor circuit and the MTM that describes the governing equations in (9). Comparing Fig. 4(b) to Fig. 2(b), this line conductor differs in two aspects from the equivalent circuit in Fig. 2(b) for deriving $G_{00}$ : There are two voltage sources driving the two ends of this line conductor through the two resistors, and there is a no current source input at the location $z^{\prime}$ in the $p$ th section of this line conductor.

According to the shown equivalent circuit, the eigenexpansion coefficient $t_{i j}^{\mathrm{h}}(z)$ corresponds to the voltage at the location $z$ in the $q$ th section of the line conductor. Therefore, by solving the voltage at that location, $t_{i j}^{\mathrm{h}}(z)$ results

$$
t_{00}^{\mathrm{h}}(z)=\bar{t}_{00}^{a} \bar{H}_{00}^{a}(z)+\underline{t}_{00}^{a} \underline{H}_{00}^{a}(z)
$$

where

$$
\begin{gathered}
\bar{H}_{00}^{a}(z)=\frac{\underline{Z}_{q}+Z_{q}\left(z-z_{q-1}\right)}{\underline{Z}_{q}+Z_{q} l_{q}+\bar{Z}_{q}} \\
\underline{H}_{00}^{a}(z)=\frac{\bar{Z}_{q}+Z_{q}\left(z_{q}-z\right)}{\underline{Z}_{q}+Z_{q} l_{q}+\bar{Z}_{q}} .
\end{gathered}
$$

This paper has used the same symbols as previously.

\section{Eigen-Expansion Coefficient $t_{i j}^{\mathrm{h}}(i+j>0)$}

When $i+j>0$, an equivalent TL circuit can be constructed to derive $t_{i j}^{\mathrm{h}}$. Compare (30a) to the TL equations

$$
\begin{gathered}
\frac{\partial V(z)}{\partial z}=-(R+s L) I(z) \\
\frac{\partial I(z)}{\partial z}=-(G+s C) V(z)
\end{gathered}
$$

or in an alternative form $\left(\partial^{2} V(z) / \partial z^{2}\right)-\gamma^{2} V(z)=0$. It is evident that $t_{i j}^{\mathrm{h}}(z)$ corresponds to the voltage at the location $z$ in an $n$-section nonuniform TL of propagation constant $\gamma=\gamma_{i j}$. The two ends of the TL are driven by two voltage sources $\bar{t}_{i j}^{a}$ and $\underline{t}_{i j}^{a}$ through two resistors $\bar{R}$ and $\underline{R}$. The PUL parameters of the $m$ th line section satisfy $\sqrt{R G}=\gamma_{i j}, \sqrt{R / G}=Z_{m}=$ $1 / k_{m}, L=0$, and $C=0$. The equivalent TL circuit is shown in Fig. 4(b) as well. Note that the two resistors at the two TL ends choose the resistance values enclosed in the parentheses, i.e., $\bar{R}=\gamma / \bar{h}$ and $\underline{R}=\gamma / \underline{h}$.

Since the eigen-expansion coefficient $t_{i j}^{\mathrm{h}}(z)$ corresponds to the voltage at the location $z$ in the $q$ th TL section, $t_{i j}^{\mathrm{h}}(z)$ is derived by solving the voltage at that location. The formula for $t_{i j}^{\mathrm{h}}(z)$ is given by

$$
t_{i j}^{\mathrm{h}}(z)=\bar{t}_{i j}^{a} \bar{H}_{i j}^{a}(z)+\underline{t}_{i j}^{a} \underline{H}_{i j}^{a}(z)
$$

where

$$
\begin{aligned}
& \bar{H}_{i j}^{a}(z)=\frac{\underline{Z}_{n+1}}{\bar{\xi}\left(\underline{Z}_{n+1}+\bar{R}\right)} \frac{\underline{Z}_{q} \cosh \gamma \underline{l}_{q}+Z_{q} \sinh \gamma \underline{l}_{q}}{\underline{Z}_{q} \cosh \gamma l_{q}+Z_{q} \sinh \gamma l_{q}} \\
& \underline{H}_{i j}^{a}(z)=\frac{\bar{Z}_{0}}{\underline{\xi}\left(\underline{R}+\bar{Z}_{0}\right)} \frac{\bar{Z}_{q} \cosh \gamma \bar{l}_{q}+Z_{q} \sinh \gamma \bar{l}_{q}}{\bar{Z}_{q} \cosh \gamma l_{q}+Z_{q} \cosh \gamma l_{q}}
\end{aligned}
$$

Here, $\bar{l}_{q}=z_{q}-z, \underline{l}_{q}=z-z_{q-1}$, and

$$
\begin{aligned}
& \bar{\xi}=\prod_{m=q+1}^{n}\left(\cosh \gamma l_{m}+\frac{Z_{m}}{\underline{Z}_{m}} \sinh \gamma l_{m}\right) \\
& \underline{\xi}=\prod_{m=1}^{q-1}\left(\cosh \gamma l_{m}+\frac{Z_{m}}{\bar{Z}_{m}} \sinh \gamma l_{m}\right) .
\end{aligned}
$$

In the previous procedure, the homogeneous solution $T^{\mathrm{h}}$ has been derived under the Neumann's sidewall BCs (2). When the Dirichlet's sidewall BCs (3) are imposed, the same procedure as the previous can still be followed to derive the corresponding homogeneous solution by merely changing the eigenfunction to $\phi_{i j}(x, y)=\sin (i \pi x / X) \sin (j \pi y / Y)$.

\section{Computing the Inhomogeneous Solution BY AN $\mathcal{O}(N \lg N)$ ALgORITHM}

The previous sections have derived analytical formulas for both the inhomogeneous solution $T^{\mathrm{i}}$ and the homogeneous solution $T^{\mathrm{h}}$. This section considers the fast computation of the inhomogeneous solution. Traditional Green's function-based methods often consider only the inhomogeneous solution, and compute $T^{\mathrm{i}}$ by a simple matrix-vector product operation: multiplying the power density function, given as a vector, by a matrix of Green's function values. For $N$ heat-source blocks and $N$ observation regions, such type of methods require $\mathcal{O}\left(N^{2}\right)$ computations to obtain the intended inhomogeneous temperatures. To speed up the thermal analysis of ULSI chips, this paper introduces an $\mathcal{O}(N \lg N)$ algorithm for computing 


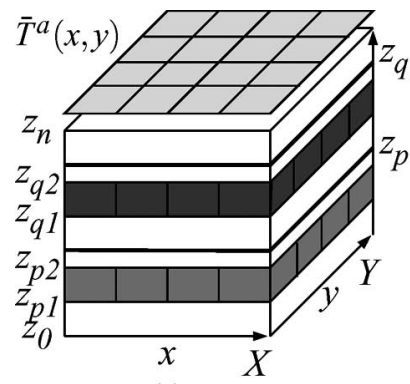

(a)

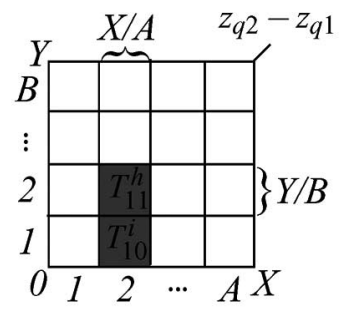

(c)

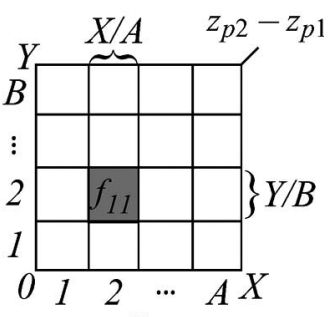

(b)

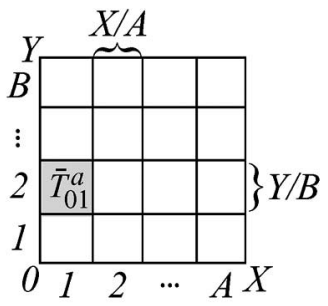

(d)

Fig. 5. Illustration of the discrete models for heat sources, homogeneous temperatures, inhomogeneous temperatures, and ambient temperatures. (a) 3-D heat-source region with $z \in\left[z_{p 1}, z_{p 2}\right]$, the target region with $z \in$ $\left[z_{q 1}, z_{q 2}\right]$, and the domain of the 2-D top ambient temperature function $\bar{T}^{a}(x, y)$. (b) Discrete heat-source model for the heat-source layer $p$. (c) Discrete homogeneous and inhomogeneous temperature models for the target layer $q$. (d) Discrete ambient temperature model for $\bar{T}^{a}(x, y)$.

the inhomogeneous solution. The algorithm for computing the homogeneous solution having the same complexity will be introduced later.

\section{A. Heat-Source Model for the Power Density Distribution $f$}

At first, the heat-source model is introduced. The proposed method employs uniform cells to discretize the heat-source regions and the target (temperature observation) regions. Heat sources in one layer, e.g., the $p$ th layer, are partitioned into $A \times$ $B$ uniform cells, each being of dimensions $(X / A) \times(Y / B) \times$ $\left(z_{p 2}-z_{p 1}\right)$ and having a uniform power density, as shown in Fig. 5(a) and (b). Inside a given layer, the cell that is the $(a+1)$ th in the $x$ direction and the $(b+1)$ th in the $y$ direction is named cell $(a, b)$, where $0 \leq a \leq A-1$ and $0 \leq b \leq B-1$. For a given cell $(a, b)$, its power density is denoted by $f_{a b}$, and its average inhomogeneous temperature is denoted by $T_{a b}^{\mathrm{i}}$.

According to (8), when there are multiple layers of heat sources, the inhomogeneous solution $T^{\mathrm{i}}$ at a given target layer, e.g., layer $q$, can be obtained by superposing each inhomogeneous solution at layer $q$ caused by a single layer of heat sources. Therefore, it is adequate by providing an algorithm that evaluates the inhomogeneous solution $T^{\mathrm{i}}$ at layer $q$, caused by the heat sources at only one layer, e.g., layer $p$. Layer $q$ is illustrated in Fig. 5(a) and (c). To obtain the inhomogeneous solution at layer $q$ caused by the heat sources in layer $p$, traditional Green's function-based methods need $\mathcal{O}\left(A^{2} B^{2}\right)$ computations, due to the dense matrix-vector product. By employing the derived multilayer heat-conduction Green's function, this paper introduces a faster yet accurate algorithm to compute the inhomogeneous solution in $\mathcal{O}(A B \lg (A B))$, which is often written as $\mathcal{O}(N \lg N)$ in this paper.

\section{B. Inhomogeneous Temperature Caused by One Layer of Heat Sources}

Consider heat-source layer $p$, which has a thickness of $z_{p 2}-$ $z_{p 1}$, as illustrated in Fig. 5(b). Insert eigen-expansion (11) into (8) and carry out that integral by convoluting the multilayer heat-conduction Green's function with the power density distribution at layer $p$. Then, the inhomogeneous temperature at an arbitrary location $(x, y, z), T^{\mathrm{i}}(x, y, z)$, is derived as follows:

$$
\begin{aligned}
& T^{\mathrm{i}}(x, y, z) \\
& =\sum_{i=0}^{\infty} \sum_{j=0}^{\infty} c_{i j} \phi_{i j}(x, y) \\
& \quad \times \int_{0}^{X} \int_{0}^{Y} \int_{z_{p 1}}^{z_{p 2}} \phi_{i j}\left(x^{\prime}, y^{\prime}\right) H_{i j}\left(z \mid z^{\prime}\right) f\left(x^{\prime}, y^{\prime}, z^{\prime}\right) d x^{\prime} d y^{\prime} d z^{\prime} \\
& =\sum_{i=0}^{\infty} \sum_{j=0}^{\infty} \frac{\sin \frac{i \pi}{2 A} \sin \frac{j \pi}{2 B}}{2^{\delta_{i 0}+\delta_{j 0}-2} i j \pi^{2}} F_{i j} \phi_{i j}(x, y) \int_{z_{p 1}}^{z_{p 2}} H_{i j}\left(z \mid z^{\prime}\right) d z^{\prime}
\end{aligned}
$$

where

$$
\begin{aligned}
F_{i j}= & \int_{0}^{X} \int_{0}^{Y} \frac{i j \pi^{2}}{X Y} \csc \frac{i \pi}{2 A} \csc \frac{j \pi}{2 B} \phi_{i j}\left(x^{\prime}, y^{\prime}\right) f\left(x^{\prime}, y^{\prime}, z^{\prime}\right) d x^{\prime} d y^{\prime} \\
= & \frac{i j \pi^{2}}{X Y} \csc \frac{i \pi}{2 A} \csc \frac{j \pi}{2 B} \\
& \times \sum_{a=0}^{A-1} \sum_{b=0}^{B-1} f_{a b} \int_{a X / A}^{(a+1) X / A(b+1) Y / B} \int_{b Y / B} \phi_{i j}\left(x^{\prime}, y^{\prime}\right) d x^{\prime} d y^{\prime} \\
= & \sum_{a=0}^{A-1} \sum_{b=0}^{B-1} 4 f_{a b} \cos \frac{i \pi(2 a+1)}{2 A} \cos \frac{j \pi(2 b+1)}{2 B} .
\end{aligned}
$$

The previous formula for $F_{i j}$ is the 2-D DCT of $f_{a b}$ [44]. Therefore, all $F_{i j}$, for $0 \leq i \leq A-1$ and $0 \leq j \leq B-1$, can be computed in $\mathcal{O}(A B \lg (A B))$ [45]. For $i, j$ outside that range, the value of $F_{i j}$ can be obtained by exploiting the periodicity of $F_{i j}: F_{(2 A-i) j}=-F_{i j}$ and $F_{i(2 B-j)}=-F_{i j}$. Note that in (36), this paper has used the following trigonometric identity in simplification:

$$
\begin{aligned}
& \int_{a X / A}^{(a+1) X / A} \int_{b X / B}^{(b+1) X / B} \phi_{i j}\left(x^{\prime}, y^{\prime}\right) d x^{\prime} d y^{\prime} \\
& =\frac{X Y}{i j \pi^{2}} \times\left[\sin \left(\frac{i \pi(2 a+1)}{2 A}+\frac{i \pi}{2 A}\right)-\sin \left(\frac{i \pi(2 a+1)}{2 A}-\frac{i \pi}{2 A}\right)\right] \\
& \quad \times\left[\sin \left(\frac{j \pi(2 b+1)}{2 B}+\frac{j \pi}{2 B}\right)-\sin \left(\frac{j \pi(2 b+1)}{B}-\frac{j \pi}{2 B}\right)\right] \\
& =\frac{4 X Y}{i j \pi^{2}} \sin \frac{i \pi}{2 A} \sin \frac{j \pi}{2 B} \cos \frac{i \pi(2 a+1)}{2 A} \cos \frac{j \pi(2 b+1)}{2 B}
\end{aligned}
$$




\section{C. $\mathcal{O}(N \lg N)$ Algorithm for Computing the Inhomogeneous Solution $T^{\mathrm{i}}$}

Consider the target layer $q$, which is of thickness $z_{q 2}-z_{q 1}$, as illustrated in Fig. 5(c). For a given cell $(a, b)$ in layer $q$, its average inhomogeneous temperature $T_{a b}^{\mathrm{i}}$ is obtained by integrating the inhomogeneous temperature $T^{\mathrm{i}}(x, y, z)$ in (35) over cell $(a, b)$, as follows:

$$
\begin{aligned}
T_{a b}^{\mathrm{i}}= & \sum_{i=0}^{\infty} \sum_{j=0}^{\infty} F_{i j} \frac{2^{2-\delta_{i 0}-\delta_{j 0}} A B \sin \frac{i \pi}{2 A} \sin \frac{j \pi}{2 B}}{i j X Y \pi^{2}\left(z_{q 2}-z_{q 1}\right)} \\
& \times \int_{z_{q 1}}^{z_{q 2} z_{z_{p 1}}} H_{i j}\left(z \mid z^{\prime}\right) d z^{\prime} d z \int_{a X / A}^{(a+1) X / A(b+1) X / B} \int_{b X / B} \phi_{i j}(x, y) d x d y \\
= & \sum_{i=0}^{\infty} \sum_{j=0}^{\infty} 2^{-\delta_{i 0}-\delta_{j 0}} F_{i j} I H_{i j} \cos \frac{i \pi(2 a+1)}{2 A} \cos \frac{j \pi(2 b+1)}{2 B}
\end{aligned}
$$

where $I H_{i j}$ is given by

$$
I H_{i j}=\frac{16 A B \sin ^{2} \frac{i \pi}{2 A} \sin ^{2} \frac{j \pi}{2 B}}{i^{2} j^{2} \pi^{4}\left(z_{q 2}-z_{q 1}\right)} \int_{z_{q 1}}^{z_{q 2}} \int_{z_{p 1}}^{z_{p 2}} H_{i j}\left(z \mid z^{\prime}\right) d z^{\prime} d z .
$$

Note that in (38), the trigonometric identify in (37) has been used.

Then, the series representation of $T_{a b}^{\mathrm{i}}$ in (38) is truncated to the following form:

$$
\begin{aligned}
T_{a b}^{\mathrm{i}} \approx \sum_{i=0}^{A-1} \sum_{j=0}^{B-1} 2^{-\delta_{i 0}-\delta_{j 0}} F_{i j} I H_{i j} \\
\quad \times \cos \frac{i \pi(2 a+1)}{2 A} \cos \frac{j \pi(2 b+1)}{2 B} .
\end{aligned}
$$

This truncated series representation of $T_{a b}^{\mathrm{i}}$ is the 2-D inverse DCT (IDCT) of $F_{i j} I H_{i j}$. As a result, the proposed algorithm to evaluate the inhomogeneous solution at layer $q$, caused by the heat sources at layer $p$, consists of one 2-D DCT procedure to compute $F_{i j}$ based on (36) and one 2-D IDCT procedure to compute $T_{a b}^{\mathrm{i}}$ based on (40). The algorithmic complexity is $\mathcal{O}(A B \lg (A B))$ [45]. Fig. 6 shows the proposed algorithm Compute- $T^{\mathrm{i}}$ for evaluating the inhomogeneous solution.

Note that the truncation of the infinite series (38) up to orders $A$ and $B$ permits one to use the 2-D DCT/IDCT to achieve an $\mathcal{O}(A B \lg (A B))$ algorithm. The accuracy of this

\section{Begin Compute- $T^{i}$}

1) Compute all $I H_{i j}$, for $0 \leq i<A$ and $0 \leq i<B$, based on (39), if they are not pre-calculated. In the pre-calculation of $I H_{i j},(41)$ and (42) are used.

2) Given one layer of heat sources, whose power densities are defined by a 2-D array made of $f_{a b}$, for $0 \leq a<A$ and $0 \leq b<B$, compute the 2-D DCT of $f_{a b}$ to obtain $F_{i j}$, based on $(36)$.

3) Form an array made of $F_{i j} I H_{i j}$, for $0 \leq i<A$ and $0 \leq i<$ $B$, and then compute the 2-D IDCT of that array to obtain $T_{a b}^{i}$, based on (40).

End Compute- $T^{i}$

Fig. 6. Compute- $T^{\mathrm{i}}: \mathcal{O}(N \lg N)$ algorithm for computing the inhomogeneous solution $T^{\mathrm{i}}$.

truncation approximation can be improved by folding back spectral components of orders higher than $A$ and $B$ into spectral components of orders lower than $A$ and $B$, i.e., adding DCT coefficients $F_{i j} I H_{i j}$ with $i \geq A$ and $j \geq B$ to the corresponding DCT coefficients $F_{i j} I H_{i j}$ with $i<A$ and $j<B$, because of the periodicity of $\cos (i \pi(2 a+1) / 2 A) \cos (j \pi(2 b+1) / 2 B)$. The later experimental results will demonstrate that the simple truncation given by (40) already provides sufficient accuracy, notwithstanding without folding back any high-order spectral components.

\section{Precharacterization of $I H_{i j}$}

For a given chip structure, all $I H_{i j}$ s should be precharacterized only once. Then, for any given power density distribution in the form of $f_{a b}$, its 2-D DCT $F_{i j}$ should be multiplied by the precalculated value of $I H_{i j}$, and then the 2-D IDCT of $F_{i j} I H_{i j}$ will produce the corresponding inhomogeneous temperature $T_{a b}^{\mathrm{i}}$. The following paragraphs detail the precharacterization of $I H_{i j}$.

Let $\hat{H}_{i j}$ denote the integral term in (39), i.e., $\hat{H}_{i j}=$ $\int_{z_{q 1}}^{z_{q 2}} \int_{z_{p 1}}^{z_{p 2}} H_{i j}\left(z \mid z^{\prime}\right) d z^{\prime} d z$. To compute $\hat{H}_{i j}$, it should be noted that in the representations of $H_{i j}$ given in (14) and (16), this paper has assumed that either the source layer $p$ is lower than the target layer $q$, or both $p=q$ and $z^{\prime} \leq z$, to simplify the presentation. When the assumption is not satisfied, $H_{i j}$ can be obtained by exploiting the reciprocity of transfer functions: If $p=q$ and $z^{\prime}>z, H_{i j}$ can be obtained by exchanging $z$ and $z^{\prime}$ in (14) and (16); otherwise, if $p>q, H_{i j}$ can be obtained by exchanging the subscripts $p$ and $q$, as well as $z$ and $z^{\prime}$, in (14) and (16). Therefore, this paper considers three cases in computing $\hat{H}_{i j}$.

1) Case That Layer $p$ and Layer $q$ are the Same $(p=q)$ : From (14) and (16), $\hat{H}_{i j}$ is obtained as shown in (41) at the bottom of the page.

$$
\hat{H}_{i j}= \begin{cases}\alpha l_{p \nu}^{2}\left[\left(\frac{\underline{Z}_{p}}{\bar{Z}_{p}}+\frac{2 z_{p 1}+z_{p 2}}{3}-z_{p-1}\right)\left(\frac{\bar{Z}_{q}}{Z_{q}}+z_{q}-\frac{2 z_{q 2}+z_{q 1}}{3}\right)-\frac{l_{q \nu}^{2}}{36}\right], & i=j=0 \\ {\left[\underline{D}_{i j}^{p}\left(e^{-\gamma l_{2 c}}-e^{-\gamma l_{1 c}}\right)^{2}+\bar{D}_{i j}^{q}\left(e^{\gamma l_{1 c}}-e^{\gamma l_{2 c}}\right)^{2}\right.} & \\ \left.+2 \underline{D}_{i j}^{p} \bar{D}_{i j}^{q} e^{-\gamma l_{q}}\left(e^{\gamma l_{q \nu}}-\gamma l_{q \nu}-1\right)+2 e^{\gamma l_{p}}\left(e^{-\gamma l_{p \nu}}+\gamma l_{p \nu}-1\right)\right] E_{i j}, & i+j>0\end{cases}
$$


Here, $l_{p \nu}=z_{p 2}-z_{p 1}, l_{q \nu}=z_{q 2}-z_{q 1}, l_{1 c}=z_{p 1}-\left(z_{p}+\right.$ $\left.z_{p-1} / 2\right)$, and $l_{2 c}=z_{p 2}-\left(z_{p}+z_{p-1} / 2\right)$.

2) Case That $p<q$ : From (14) and (16), $\hat{H}_{i j}$ is obtained as follows:

$$
\hat{H}_{i j}=\left\{\begin{array}{rlrl}
\alpha l_{p \nu} l_{q \nu}\left(\frac{\underline{Z}_{p}}{Z_{p}}+\frac{z_{p 1}+z_{p 2}}{2}-z_{p-1}\right) & \\
\quad \times\left(\frac{\bar{Z}_{q}}{Z_{q}}+z_{q}-\frac{z_{q 1}+z_{q 2}}{2}\right), & i=j=0 \\
E_{i j}\left[\underline{D}_{i j}^{p}\left(e^{-\gamma \underline{l}_{p 1}}-e^{-\gamma \underline{l}_{p 2}}\right)+e^{\gamma \underline{l}_{p 2}}-e^{\gamma \underline{l}_{p 1}}\right] & \\
& \times\left[\bar{D}_{i j}^{q}\left(e^{-\gamma \bar{l}_{q 2}}-e^{-\gamma \bar{l}_{q 1}}\right)+e^{\gamma \bar{l}_{q 1}}-e^{\gamma \bar{l}_{q 2}}\right], & & i+j>0 .
\end{array}\right.
$$

Here, $\underline{l}_{p 1,2}=z_{p 1,2}-z_{p-1}$ and $\bar{l}_{q 1,2}=z_{q}-z_{q 1,2}$.

3) Case That $p>q$ : The formula for $\hat{H}_{i j}$ is similar to (42). In this case, $\hat{H}_{i j}$ can be obtained by exchanging the subscripts $p$ and $q$ in (42), and also the subscripts $p$ and $q$ in coefficients $\alpha, \underline{D}_{i j}^{p}, \bar{D}_{i j}^{q}$, and $E_{i j}$.

The previously used coefficients are given by

$$
\begin{aligned}
\alpha & =\frac{Z_{p} Z_{q}}{\underline{Z}_{p}+Z_{p} l_{p}+\bar{Z}_{q}} \\
\underline{D}_{i j}^{p} & =\frac{\underline{Z}_{p}-Z_{p}}{\underline{Z}_{p}+Z_{p}} \\
\bar{D}_{i j}^{q} & =\frac{\bar{Z}_{q}-Z_{q}}{\bar{Z}_{q}+Z_{q}} \\
E_{i j} & =\frac{\xi}{4 \gamma^{2}} \frac{\left(\underline{Z}_{p}+Z_{p}\right)\left(\bar{Z}_{q}+Z_{q}\right)}{Z_{p}\left(\underline{Z}_{p}+\bar{Z}_{p}\right) \cosh \gamma l_{p}+\left(Z_{p}^{2}+\underline{Z}_{p} \bar{Z}_{p}\right) \sinh \gamma l_{p}} .
\end{aligned}
$$

According to the equivalent TL circuit shown in Fig. 1(d), $\underline{D}_{i j}^{p}$ is the reflection coefficient of the $p$ th TL section, seen from its bottom boundary toward the bottom side of the circuit, and $\bar{D}_{i j}^{q}$ is the reflection coefficient of the $q$ th TL section, seen from its top boundary toward the top side of the circuit.

The coefficients $\underline{D}_{i j}^{p}, \bar{D}_{i j}^{q}$, and $E_{i j}$ depend upon a single parameter $\gamma$, where $\gamma=\sqrt{\left(i^{2} \pi^{2} / X^{2}\right)+\left(j^{2} \pi^{2} / Y^{2}\right)}$. For a given process technology, therefore, regardless of $A$ and $B$, these coefficients can be characterized into one-dimensional (1-D) lookup tables indexed by the parameter $\gamma$. Then, in the precharacterization of $I H_{i j}$ for the given values of $A$ and $B$, the required values of $\underline{D}_{i j}^{p}, \bar{D}_{i j}^{q}$, and $E_{i j}$ can be obtained from those 1-D lookup tables, and then $I H_{i j}$ can be simply calculated from (39), (41), and (42).

\section{Computing the Homogeneous Solution By AN $\mathcal{O}(N \lg N)$ Algorithm}

Section $\mathrm{V}$ has given a set of analytical formulas for the homogeneous solution $T^{\mathrm{h}}$. Based on the given formulas, this section introduces an $\mathcal{O}(N \lg N)$ algorithm for computing $T^{\mathrm{h}}$. The introduced algorithm decomposes a target region in a layer (say, layer $q$ ) of vertical dimension spanning $\left[z_{q 1}, z_{q 2}\right]$ into $A \times B$ uniform cells, as illustrated by Fig. 5(a) and (c) again. Similarly, the algorithm partitions the domains of the top and bottom 2-D ambient temperature functions $\bar{T}^{a}(x, y)$ and $\underline{T}^{a}(x, y)$ into $A \times B$ uniform cells, as illustrated by Fig. 5(a) and (d). To simplify notations, this paper still adopts $A$ and $B$ to denote the number of cells in the $x$ and $y$ directions, respectively, even though the number of cells employed in calculating the homogeneous solution can be different from that in calculating the inhomogeneous solution. Each cell in a target region is of dimensions $(X / A) \times(Y / B) \times\left(z_{q 2}-z_{q 1}\right)$ and has a uniform homogeneous temperature, as shown in Fig. 5(c). Each cell discretizing the domains of the top and bottom ambient temperature functions is of dimensions $(X / A) \times(Y / B)$ and has a uniform ambient temperature, as shown in Fig. $5(d)$. For a given cell $(a, b)$ in layer $q$, its average homogeneous temperature is denoted by $T_{a b}^{\mathrm{h}}$, and its overall temperature is denoted by $T_{a b}$, which is given by $T_{a b}=T_{a b}^{\mathrm{i}}+T_{a b}^{\mathrm{h}}$. For a given cell $(a, b)$ that discretizes the domain of an ambient temperature function, its ambient temperature is denoted by $\bar{T}_{a b}^{a}$ or $\underline{T}_{a b}^{a}$, depending on which ambient temperature function the cell represents.

\section{A. Eigen-Expansion Coefficient $\bar{t}_{i j}^{a}$ and $\underline{t}_{i j}^{a}$}

The eigen-expansion coefficient $\bar{t}_{i j}^{a}$ in the eigen-expansion $(29 b)$ is defined by an integral

$$
\bar{t}_{i j}^{a}=\frac{2^{2-\delta_{i 0}-\delta_{j 0}}}{X Y} \int_{0}^{X} \int_{0}^{Y} \bar{T}^{a}(x, y) \phi_{i j}(x, y) d x d y .
$$

Use the introduced discretization scheme and carry out the above integral to reformulate $\bar{t}_{i j}^{a}$ as follows:

$$
\begin{aligned}
\bar{t}_{i j}^{a}= & \frac{2^{2-\delta_{i 0}-\delta_{j 0}}}{X Y} \sum_{a=0}^{A-1} \sum_{b=0}^{B-1} \bar{T}_{a b}^{a} \\
& \times \int_{a X / A} \int_{b X / B}^{(a+1) X / A(b+1) X / B} \phi_{i j}(x, y) d x d y \\
= & \frac{4}{i j \pi^{2} 2^{\delta_{i 0}+\delta_{j 0}}} \sin \left(\frac{i \pi}{2 A}\right) \sin \left(\frac{j \pi}{2 B}\right) \bar{t}_{i j}^{n}
\end{aligned}
$$

where $\bar{t}_{i j}^{n}$ is given by

$$
\bar{t}_{i j}^{n}=\sum_{a=0}^{A-1} \sum_{b=0}^{B-1} 4 \bar{T}_{a b}^{a} \cos \frac{i \pi(2 a+1)}{2 A} \cos \frac{j \pi(2 b+1)}{2 B} .
$$

In the formulation of $\bar{t}_{i j}^{a}$ in (43), this paper again used the trigonometric identity (37).

Similarly, based on (29c), the eigen-expansion coefficient $\underline{t}_{i j}^{a}$ is represented as

$$
\underline{t}_{i j}^{a}=\frac{2^{2-\delta_{i 0}-\delta_{j 0}}}{i j \pi^{2}} \sin \left(\frac{i \pi}{2 A}\right) \sin \left(\frac{j \pi}{2 B}\right) \underline{t}_{i j}^{n}
$$


where $\underline{t}_{i j}^{n}$ is given by

$$
\underline{t}_{i j}^{n}=\sum_{a=0}^{A-1} \sum_{b=0}^{B-1} 4 \underline{T}_{a b}^{a} \cos \frac{i \pi(2 a+1)}{2 A} \cos \frac{j \pi(2 b+1)}{2 B} .
$$

Formulas (44) and (46) correspond to the 2-D DCTs of the top and bottom ambient temperatures $\bar{T}_{a b}^{a}$ and $\underline{T}_{a b}^{a}$. Therefore, all $\bar{t}_{i j}^{n}$ and $\underline{t}_{i j}^{n}$, for $0 \leq i \leq A-1$ and $0 \leq j \leq B-1$, can be computed in $\mathcal{O}(A B \lg (A B))$.

\section{B. $\mathcal{O}(N \lg N)$ Algorithm for Evaluating the Homogeneous Solution $T^{\mathrm{h}}$}

For a given cell $(a, b)$ in the target region, its average homogeneous temperature $T_{a b}^{\mathrm{h}}$ is obtained by integrating $T^{\mathrm{h}}(x, y, z)$ in (29a) over cell $(a, b)$, as follows:

$$
\begin{aligned}
T_{a b}^{\mathrm{h}}= & \frac{A B}{X Y\left(z_{q 2}-z_{q 1}\right)} \\
& \times \sum_{i=0}^{\infty} \sum_{j=0}^{\infty} \int_{a X / A}^{(a+1) X / A} \int_{b X / B}^{(b+1) X / B} \int_{z_{q 1}}^{z_{q 2}} \phi_{i j}(x, y) t_{i j}^{\mathrm{h}}(z) d x d y d z \\
= & \sum_{i=0}^{\infty} \sum_{j=0}^{\infty} 2^{-\delta_{i 0}-\delta_{j 0}} \mathrm{TH}_{i j} \cos \frac{i \pi(2 a+1)}{2 A} \cos \frac{i \pi(2 b+1)}{2 B} .
\end{aligned}
$$

Here, $\mathrm{TH}_{i j}$ is given by

$$
\begin{aligned}
\mathrm{TH}_{i j} & =\frac{2^{2+\delta_{i 0}+\delta_{j 0}} A B \sin \left(\frac{i \pi}{2 A}\right) \sin \left(\frac{j \pi}{2 B}\right)}{i j \pi^{2}\left(z_{q 2}-z_{q 1}\right)} \int_{z_{q 1}}^{z_{q 2}} t_{i j}^{\mathrm{h}}(z) d z \\
& =\frac{16 A B \sin ^{2} \frac{i \pi}{2 A} \sin ^{2} \frac{j \pi}{2 B}}{i^{2} j^{2} \pi^{4}\left(z_{q 2}-z_{q 1}\right)}\left[\bar{t}_{i j}^{n} \overline{I H}_{i j}^{a}+\underline{t}_{i j}^{n} \underline{H}_{i j}^{a}\right]
\end{aligned}
$$

where $\overline{I H}_{i j}^{a}$ and $\underline{I H}_{i j}^{a}$ are the integrals of $\bar{H}_{i j}^{a}$ and $\underline{H}_{i j}^{a}$ over interval $\left[z_{q 1}, z_{q 2}\right]$, i.e.

$$
\overline{I H}_{i j}^{a}=\int_{z_{q 1}}^{z_{q 2}} \bar{H}_{i j}^{a}(z) d z \text { and } \underline{I H}_{i j}^{a}=\int_{z_{q 1}}^{z_{q 2}} \underline{H}_{i j}^{a}(z) d z .
$$

Then, the series representation of $T_{a b}^{\mathrm{h}}$ in (47) is truncated to the following form:

$$
T_{a b}^{\mathrm{h}} \approx \sum_{i=0}^{A-1} \sum_{j=0}^{B-1} 2^{-\delta_{i 0}-\delta_{j 0}} \mathrm{TH}_{i j} \cos \frac{i \pi(2 a+1)}{2 A} \cos \frac{i \pi(2 b+1)}{2 B}
$$

which corresponds to the 2-D IDCT of $\mathrm{TH}_{i j}$. Therefore, all $T_{a b}^{\mathrm{h}}$, for $0 \leq a \leq A-1$ and $0 \leq b \leq B-1$, can be computed in $\mathcal{O}(A B \lg (A B))$. Based on the formulas derived previously, an $\mathcal{O}(A B \lg (A B))$ algorithm, named Compute- $T^{\mathrm{h}}$, is obtained for computing the homogeneous solution: First compute the 2-D DCTs of the discrete top and bottom ambient temperatures $\bar{T}_{a b}^{a}$ and $\underline{T}_{a b}^{a}$ to obtain $\bar{t}_{i j}^{n}$ and $\underline{t}_{i j}^{n}$, based on (44) and (46);
Begin Compute- $T^{h}$

1) Based on (44) and (46), compute the 2-D DCT's of the given top and bottom ambient temperatures $T_{a b}^{a}$ and $T_{a b}^{a}$ to obtain $\bar{t}_{i j}^{n}$ and $\underline{t}_{i j}^{n}$.

2) Compute all $T H_{i j}$, for $0 \leq i \leq A-1$ and $0 \leq j \leq B-1$, based on (48). In the calculation of $T H_{i j},(51)$ and (52) are used.

3) Based on (50), compute the 2-D IDCT of $T H_{i j}$ to obtain $T_{a b}^{h}$. End Compute- $T^{h}$

Begin LOTAGre

1) Employ algorithm Compute- $T^{i}$ to obtain $T_{a b}^{i}$

2) Employ algorithm Compute- $T^{h}$ to obtain $T_{a b}^{k}$.

3) Add $T_{a b}^{i}$ and $T_{a b}^{h}$ to obtain temperature $T_{a b}$.

\section{End LOTAGre}

Fig. 7. LOTAGre: $\mathcal{O}(N \lg N)$ thermal-analysis method based on the multilayer heat-conduction Green's function.

then compute all $\mathrm{TH}_{i j}$, for $0 \leq i \leq A-1$ and $0 \leq j \leq B-1$, according to (48); finally compute the 2-D IDCT of $\mathrm{TH}_{i j}$ to obtain $T_{a b}^{\mathrm{h}}$, based on (50). The complete thermal-analysis method LOTAGre, which integrates the $\mathcal{O}(N \lg N)$ algorithms Compute- $T^{\mathrm{i}}$ and Compute- $T^{\mathrm{h}}$, is shown in Fig. 7.

Note that folding back high-order spectral components $\mathrm{TH}_{i j}$ s can also improve the accuracy of the truncation approximation (50). However, the later experimental results will demonstrate that the simple truncation up to order $A$ and $B$, (50) is sufficiently accurate notwithstanding without the folding back of any high-order spectral components.

\section{Formulas for $\overline{I H}_{i j}^{a}$ and $\underline{I H}_{i j}^{a}$}

Based on (32) and (34), $\overline{I H}_{i j}^{a}$ and $\underline{I H}_{i j}^{a}$ in (49) are derived in the following.

For $i+j=0$

$$
\begin{aligned}
\overline{I H}_{00}^{a}= & \frac{\underline{Z}_{q} l_{q \nu}+Z_{q} l_{q \nu} \underline{l}_{q 12 c}}{\underline{Z}_{q}+Z_{q} l_{q}+\bar{Z}_{q}} \\
\underline{I H}_{00}^{a}= & \frac{\bar{Z}_{q} l_{q \nu}+Z_{q} l_{q \nu} \bar{l}_{q 12 c}}{\underline{Z}_{q}+Z_{q} l_{q}+\bar{Z}_{q}}
\end{aligned}
$$

where $\underline{l}_{q 12 c}=\left(z_{q 2}+z_{q 1} / 2\right)-z_{q-1}$ and $\bar{l}_{q 12 c}=z_{q}-\left(z_{q 2}+\right.$ $\left.z_{q 1} / 2\right)$.

For $i+j>0$

$$
\begin{aligned}
\overline{I H}_{i j}^{a}= & \frac{\underline{Z}_{q}+Z_{q}}{2 \gamma \bar{\xi}\left(1+\bar{R} / \underline{Z}_{n+1}\right)} \\
& \times \frac{e^{\gamma \underline{l}_{q 2}}-e^{\gamma \underline{l}_{q 1}}+\underline{D}_{i j}^{q}\left(e^{-\gamma \underline{l}_{q 1}}-e^{-\gamma \underline{l}_{q 2}}\right)}{\underline{Z}_{q} \cosh \gamma l_{q}+Z_{q} \sinh \gamma l_{q}} \\
\underline{I H}_{i j}^{a}= & \frac{\bar{Z}_{q}+Z_{q}}{2 \gamma \underline{\xi}\left(1+\underline{R} / \bar{Z}_{0}\right)} \\
& \times \frac{e^{\gamma \bar{l}_{q 1}}-e^{\gamma \bar{l}_{q 2}}+\bar{D}_{i j}^{q}\left(e^{-\gamma \bar{l}_{q 2}}-e^{-\gamma \bar{l}_{q 1}}\right)}{\bar{Z}_{q} \cosh \gamma l_{q}+Z_{q} \sinh \gamma l_{q}}
\end{aligned}
$$

where $\underline{l}_{q 1,2}=z_{q 1,2}-z_{q-1}$, and $\underline{D}_{i j}^{q}$ is the reflection coefficient of the $q$ th TL section, seen from its bottom boundary toward 


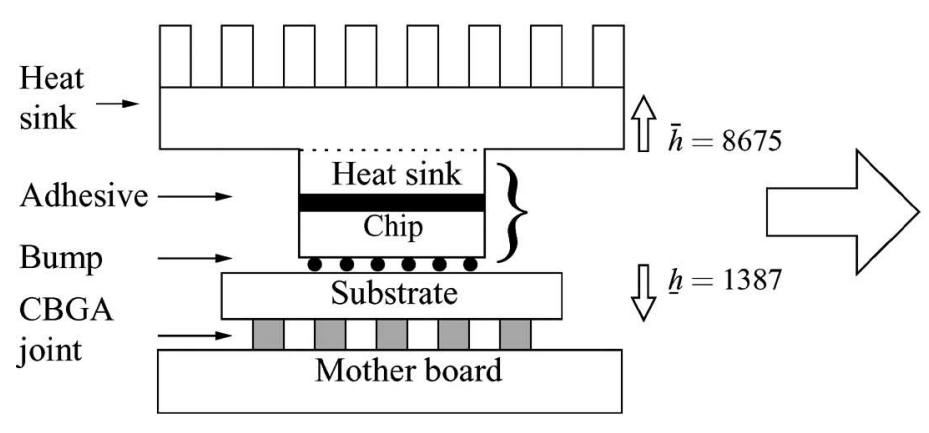

(a)

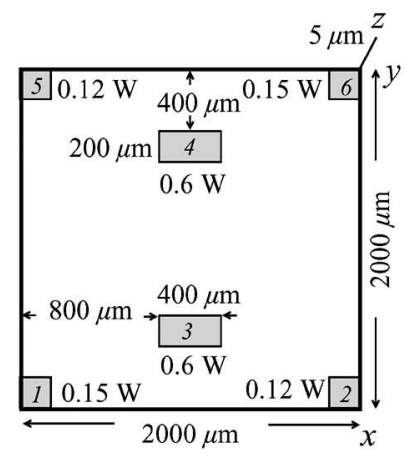

(c)

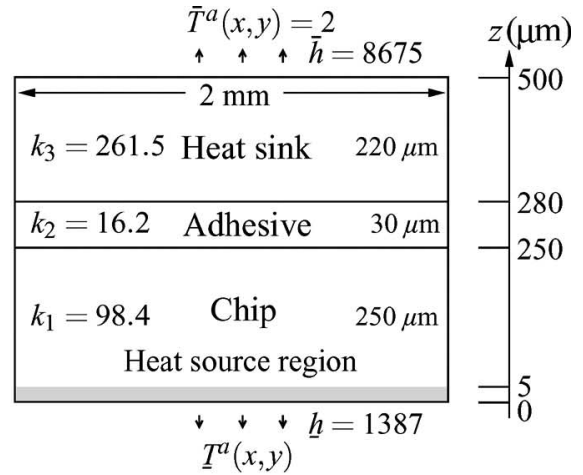

(b)

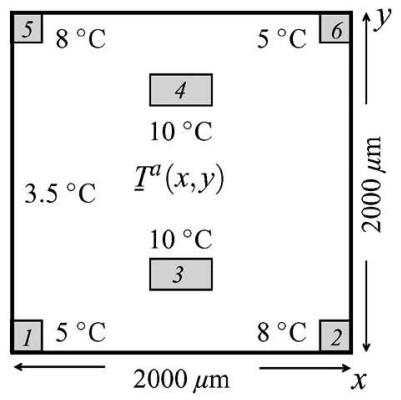

(d)

Fig. 8. Example chip of flip-chip packaging. (a) Real chip structure. (b) MTM for the given chip. (c) Specification of the power density distribution $f$ for the heat-source region. (d) Specification of the 2-D bottom ambient temperature function $\underline{T}^{a}(x, y)$, which models the thermal effects of the bumps.

the bottom side of the equivalent TL circuit, i.e., $\underline{D}_{i j}^{q}=\left(\underline{Z}_{q}-\right.$ $\left.Z_{q} / \underline{Z}_{q}+Z_{q}\right)$. Since $\overline{I H}_{i j}^{a}$ and $\underline{I H} \underline{H}_{i j}^{a}$ are functions of a single parameter $\gamma$, where $\gamma=\sqrt{\left(i^{2} \pi^{2} / X^{2}\right)+\left(j^{2} \pi^{2} / Y^{2}\right)}$, 1-D lookup tables can also be used to speed up the computing of the two integrals.

Both Compute- $T^{\mathrm{i}}$ and Compute- $T^{\mathrm{h}}$ use the 2-D DCT and 2-D IDCT procedures to achieve an $\mathcal{O}(N \lg N)$ complexity. However, the involved DCT and IDCT coefficients have different physical meanings. In Compute- $T^{\mathrm{i}}, I H_{i j}$ is a major coefficient, which is related to the transfer impedance of the equivalent circuits. In Compute- $T^{\mathrm{h}}, \overline{I H}_{i j}$ and $\underline{I H} i j$ are major coefficients however, which are related to the voltage transfer functions of the equivalent circuits.

\section{EXPERIMENTAL RESULTS}

\section{A. Example Chip}

The proposed $\mathcal{O}(N \lg N)$ thermal-analysis method based on the multilayer heat-conduction Green's function, named LOTAGre, has been verified by comparisons with a CFD tool, called FLUENT. As introduced in Section II, by employing the MTM in Fig. 1(b), LOTAGre can consider different types of chip packaging scenarios, e.g., wire-bonding packaging and flip-chip packaging. The following uses a die in flip-chip packaging to demonstrate LOTAGre. Fig. 8 shows the chip example, which has a structure similar to the PowerPC chip [12], [36].

The figure shows two heat-conduction paths for the flip-chip packaged chip. One heat-conduction path transfers the heat generated in the chip active region through the silicon bulk, the thermal adhesive, and the heat sink to the top ambient environment. The other heat-conduction path transfers the heat through the bump, the under-fill materials, and the substrate to the bottom ambient environment. Fig. 8(b) shows a threelayer MTM for modeling the example chip. In the MTM, the bottom two layers incorporate the entire chip and the thermal adhesive, and the top layer models one portion of the heat sink. The thermal effects of the other regions excluded in the MTM are addressed by the top heat transfer rate $\bar{h}$, the bottom heat transfer rate $\underline{h}$, and the top and bottom ambient temperature functions $\bar{T}^{a}(x, y)$ and $\underline{T}^{a}(x, y)$. The two heat transfer rates $\bar{h}$ and $\underline{h}$ can be determined by either empirical formulas or experimental data fitting [34], [36]. In this paper, $\bar{h}$ is set to $8675 \mathrm{~W} /\left(\mathrm{m}^{2} \cdot \mathrm{K}\right)$ and $\underline{h}$ is set to $1387 \mathrm{~W} /\left(\mathrm{m}^{2} \cdot \mathrm{K}\right)$. The chip horizontal dimensions are $2 \times 2 \mathrm{~mm}^{2}$. Fig. 8(b) also shows the thickness and thermal conductivity of each layer. Inside the chip layer, there is a 5- $\mu \mathrm{m}$ thick heat-source region, where six rectangular heat sources are placed. Fig. 8(c) shows the six heat sources, and the power of each heat source is given near the related heat-source box.

The temperature distribution of the example chip was analyzed using LOTAGre and FLUENT. In using LOTAGre, $A$ and $B$ were set to 40 . However, it is recommended that $A$ and $B$ be the powers of two, for facilitating the DCT and IDCT procedures. At first, the homogeneous temperature distribution was analyzed. The 2-D top ambient temperature function $\bar{T}^{a}(x, y)$ was assumed being a constant function. The specified 2-D bottom ambient temperature function $\underline{T}^{a}(x, y)$ is shown 

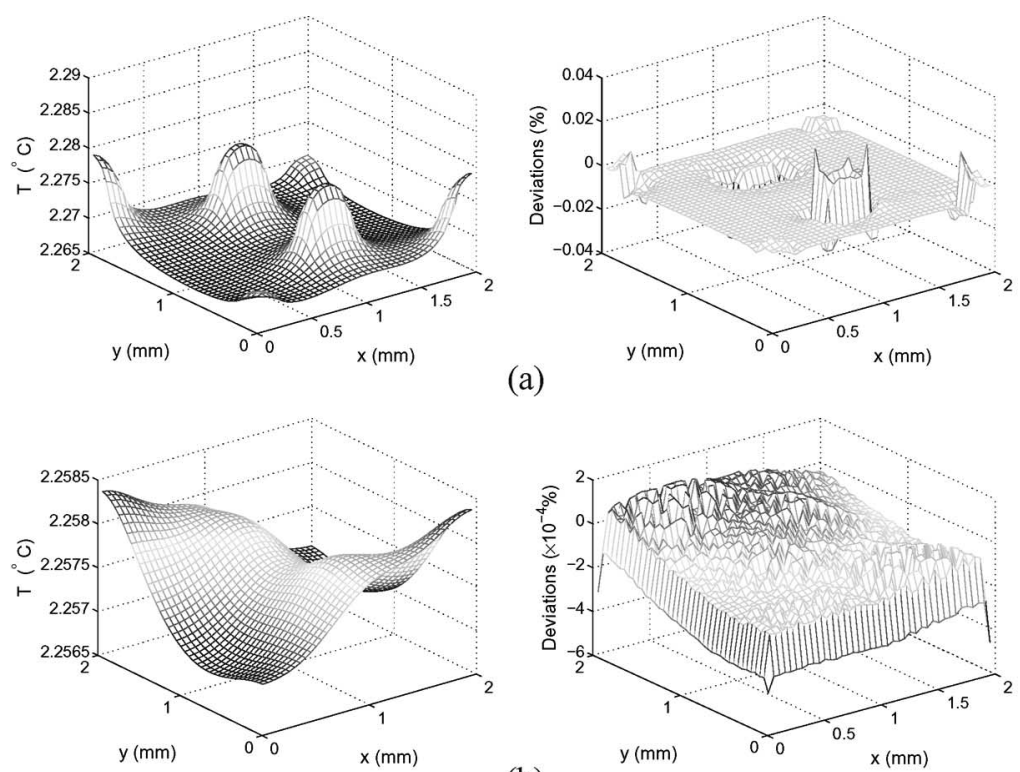

(a)
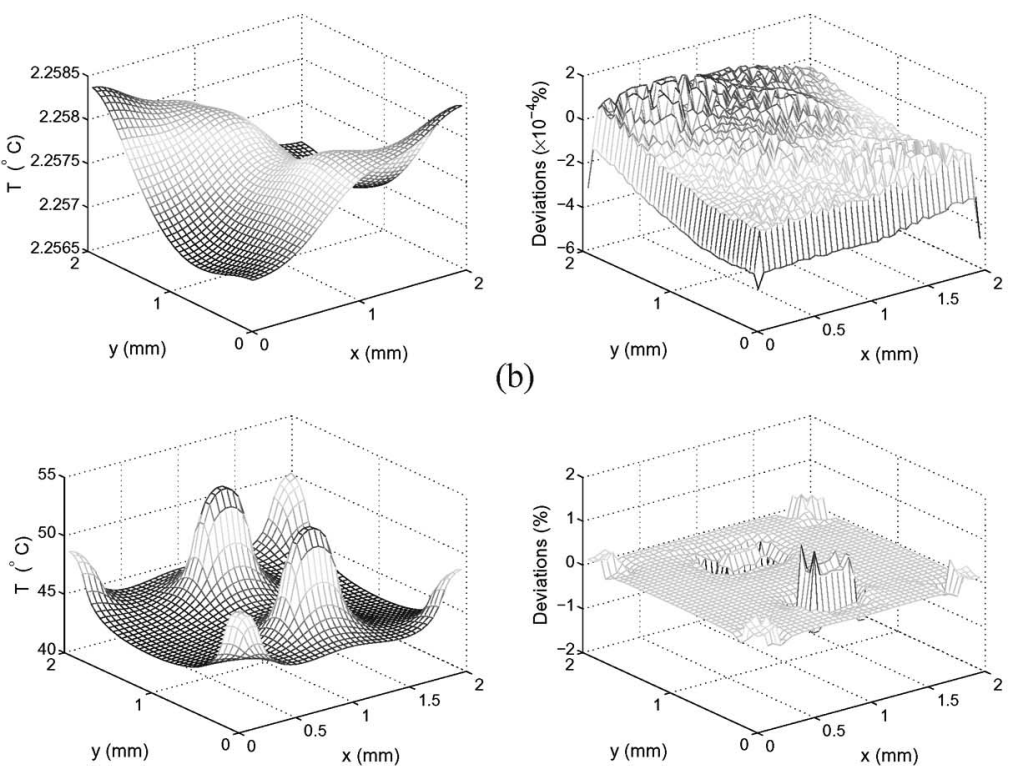

(c)
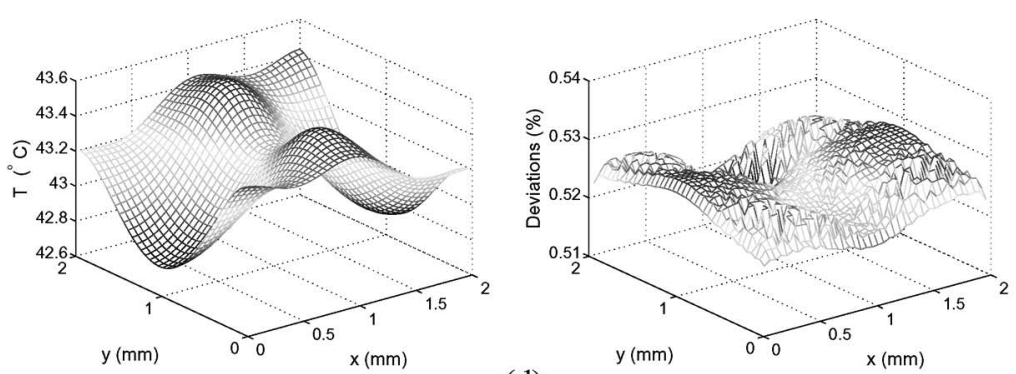

(d)

Fig. 9. Comparisons between LOTAGre and FLUENT in computing the homogeneous and inhomogeneous temperature distributions. In (a), (b), (c), and (d), the left graphs show the temperature distributions computed by LOTAGre, and the right graphs show the relative temperature differences from FLUENT in percentages. (a) Homogeneous temperature distribution in the heat-source region. (b) Homogeneous temperature distribution on the top surface of the MTM. (c) Inhomogeneous temperature distribution in the heat-source region. (d) Inhomogeneous temperature distribution on the top surface of the MTM.

in Fig. 8(d). As shown by Fig. 8(c) and (d), the specified bottom ambient temperature function was very similar to the specified power density function, except that the ambient temperature values replaced the power values in Fig. 8(c).

The results for the homogeneous temperature distribution are shown in Fig. 9(a) and (b), where the left graphs give the homogeneous temperature distributions obtained from LOTAGre, and the right graphs show the relative differences of the calculated temperatures from FLUENT. Fig. 9(a) and (b) demonstrates that the homogeneous temperature deviations between the two methods are within $0.04 \%$ for the heat-source region and within $0.001 \%$ for the top surface of the MTM. In terms of CPU usage, LOTAGre took $1.193 \mathrm{~s}$ to precharacterize all $\overline{I H}_{i j}$ and $\underline{I H}_{i j}$ and then took only $47 \mathrm{~ms}$ to calculate the homogeneous temperature distribution, while FLUENT took $269 \mathrm{~s}$ to obtain the solution. A SUN Blade 1500 machine was used in running the experiments.

Fig. 9(c) and (d) shows the results for the inhomogeneous temperature distribution. The left graphs give the inhomogeneous temperature distributions obtained by LOTAGre, and the right graphs show the relative temperature differences from FLUENT. Fig. 9(c) and (d) again demonstrates the accuracy of LOTAGre: The inhomogeneous temperature deviations between the two methods are within $1.18 \%$ for the heat-source region and within $0.529 \%$ for the top surface of the MTM. The precharacterization of $I H_{i j}$ took $1.283 \mathrm{~s}$. The evaluation of the 
TABLE I

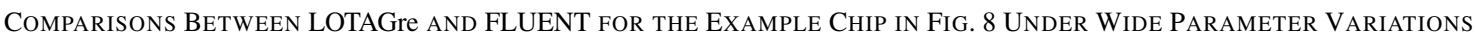

\begin{tabular}{|c|c|c|c|c|c|c|c|c|c|c|c|c|}
\hline $\begin{array}{c}\underline{h} \\
\left(\mathrm{~W} / \mathrm{m}^{2} \mathrm{~K}\right)\end{array}$ & $\begin{array}{c}k_{2} \\
(\mathrm{~W} / \mathrm{m} \mathrm{K})\end{array}$ & $\begin{array}{c}h \\
\left(\mathrm{~W} / \mathrm{m}^{2} \mathrm{~K}\right)\end{array}$ & $\begin{array}{c}\text { H-Max. } \\
\left({ }^{\circ} \mathrm{C}\right)\end{array}$ & $\begin{array}{l}\text { H-Pre. } \\
\text { (s) }\end{array}$ & $\begin{array}{c}H-E v a . \\
(\mathrm{ms})\end{array}$ & $\begin{array}{c}H-F L U . \\
(\mathrm{s})\end{array}$ & H-Dev. & $\begin{array}{c}\text { I-Max. } \\
\left({ }^{\circ} \mathrm{C}\right)\end{array}$ & $\begin{array}{l}\text { I-Pre. } \\
\text { (s) }\end{array}$ & $\begin{array}{l}\text { I-Eva. } \\
(\mathrm{ms})\end{array}$ & $\begin{array}{c}I-F L U . \\
(\mathrm{s})\end{array}$ & I-Dev. \\
\hline 257 & 12.2 & 8675 & 2.058 & 1.238 & 52 & 290 & $0.02 \%$ & 59.44 & 1.215 & 42 & 51 & $0.95 \%$ \\
\hline 5212 & 32.4 & 5419 & 2.968 & 1.203 & 45 & 83 & $0.59 \%$ & 49.51 & 1.266 & 42 & 182 & $1.27 \%$ \\
\hline 9826 & 16.2 & 2371 & 3.587 & 1.17 & 57 & 134 & $0.74 \%$ & 43.94 & 1.327 & 46 & 202 & $1.44 \%$ \\
\hline 1387 & 5.1 & 9451 & 2.269 & 1.14 & 47 & 166 & $0.05 \%$ & 51.71 & 1.255 & 48 & 373 & $1.23 \%$ \\
\hline 2715 & 382 & 7213 & 2.542 & 1.211 & 48 & 93 & $0.26 \%$ & 52.51 & 1.226 & 53 & 34 & $1.16 \%$ \\
\hline 1462 & 1715 & 8415 & 2.295 & 1.187 & 47 & 291 & $0.04 \%$ & 52.88 & 1.214 & 43 & 140 & $1.16 \%$ \\
\hline 512 & 10.4 & 800.1 & 2.74 & 1.197 & 50 & 941 & $0.38 \%$ & 340.9 & 1.238 & 42 & 553 & $0.55 \%$ \\
\hline 1128 & 15.3 & 3410 & 2.482 & 1.154 & 51 & 238 & $0.85 \%$ & 105.5 & 1.32 & 46 & 232 & $0.55 \%$ \\
\hline 1682 & 60.1 & 13215 & 2.233 & 1.171 & 48 & 145 & $0.07 \%$ & 38.58 & 1.27 & 42 & 364 & $1.67 \%$ \\
\hline 21 & 70.2 & 9898 & 2.004 & 1.195 & 48 & 223 & $0.01 \%$ & 53.56 & 1.227 & 45 & 81 & $1.17 \%$ \\
\hline
\end{tabular}

inhomogeneous temperature distribution after the precharacterization took only $44 \mathrm{~ms}$, while FLUENT took $205 \mathrm{~s}$ to obtain the solution.

Different sets of parameters $\underline{h}, k_{2}$, and $\bar{h}$ were also experimented for the MTM shown in Fig. 8(b), and the temperature distributions of the top surface and the heat-source region of the MTM were again solved by LOTAGre and FLUENT, respectively. The results are shown in Table I, where letter $H$ indicates the homogeneous temperature results, while letter $I$ indicates the inhomogeneous temperature results; Max. indicates the maximum temperature; Pre. indicates the precharacterization time taken by LOTAGre; Eva. indicates the temperature evaluation time taken by LOTAGre; FLU. indicates the run time of FLUENT; and Dev. indicates the maximum of the relative temperature differences from FLUENT. The results in the table have demonstrated the accuracy of LOTAGre, despite the large parameter variations. They also demonstrate the superior speed advantage of LOTAGre, which is around two orders of magnitude faster than FLUENT if the precharacterization time is taken into account. If LOTAGre is used in an inner loop and iterated many times for different power density distributions, the precharacterization of coefficients should be conducted only once. Therefore, LOTAGre can asymptotically be thousands of times faster than FLUENT. Table I shows that the homogeneous temperature differences between LOTAGre and FLUENT are within $0.9 \%$, and the inhomogeneous temperature differences between the two methods are within $1.7 \%$.

\section{B. Scalability of LOTAGre}

Theoretically, LOTAGre has an $\mathcal{O}(N \lg N)$ complexity, while the traditional Green's function-based thermal-analysis methods are of quadratic complexity [14]-[16]. To demonstrate the scalability, LOTAGre was employed to analyze the example chip in Fig. 8 after extending the $x-y$ dimensions to $1.28 \times$ $1.28 \mathrm{~cm}^{2}$ to accommodate more standard cells. A randomly generated heat-source distribution $f_{a b}$ was imposed on the $5-\mu \mathrm{m}$ thick heat-source region of the chip, which is indicated in Fig. 8(b). Only the inhomogeneous temperature distribution was analyzed, since the homogeneous temperature distribution does not depend on the power density distribution and can be computed only once for a given ambient condition. The heatsource power density distribution and the calculated inhomo-

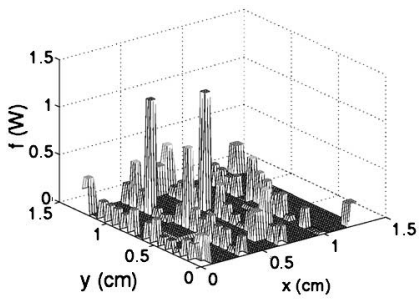

(a)

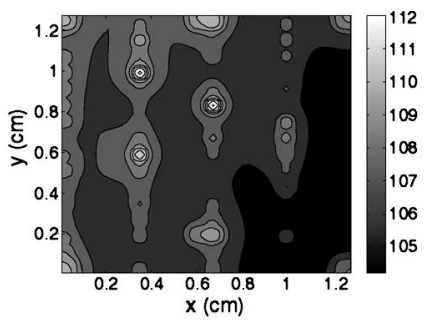

(b)

\begin{tabular}{|c|c||c||c|c|}
\hline$A$ & $B$ & Pre-char. $(\mathrm{s})$ & LOTAGre $(\mathrm{s})$ & Trad. $(\mathrm{s})$ \\
\hline 32 & 32 & 0.038 & 0.021 & 0.012 \\
\hline 32 & 64 & 0.073 & 0.023 & 0.046 \\
\hline 64 & 64 & 1.442 & 0.026 & 0.192 \\
\hline 64 & 128 & 2.866 & 0.032 & 0.85 \\
\hline 128 & 128 & 5.825 & 0.047 & 4.733 \\
\hline 128 & 256 & 11.501 & 0.082 & 18.75 \\
\hline 256 & 256 & 22.713 & 0.171 & 82.892 \\
\hline 256 & 512 & 45.663 & 0.407 & 707.07 \\
\hline 512 & 512 & 90.79 & 1.236 & N/A \\
\hline 512 & 1024 & 181.428 & 3.282 & N/A \\
\hline 1024 & 1024 & 362.350 & 7.537 & N/A \\
\hline
\end{tabular}

Fig. 10. Scalability of LOTAGre (the table shows the CPU usage by LOTAGre and traditional Green's function-based thermal-analysis methods). (a) Randomly generated heat source distribution $f$. (b) $T^{i}$ in the heat source region (units in ${ }^{\circ} \mathrm{C}$ ).

geneous temperature distribution of the heat-source region are shown in Fig. 10(a) and (b).

The CPU usage taken by LOTAGre during precharacterization is shown in the table in Fig. 10 (Pre-char.), for the number of cells $A \times B$ varying from $32 \times 32$ to $1024 \times 1024$. Note that the CPU usage during precharacterization will be amortized in an iterative thermal-analysis flow, since LOTAGre conducts the precharacterization of $I H_{i j}$ only once. The table shows that the precharacterization time almost linearly related to $A \times B$, as all $I H_{i j}$ for $0 \leq i \leq A-1$ and $0 \leq j \leq B-1$ were computed by LOTAGre. As introduced in Section VI-D, the precharacterization time will be significantly reduced if 1-D lookup tables are established for coefficients $\underline{D}_{i j}^{p}, \bar{D}_{i j}^{q}$, and $E_{i j}$. In running the experiments in this paper, however, LOTAGre has not employed 1-D lookup tables to speed up the precharacterization. 


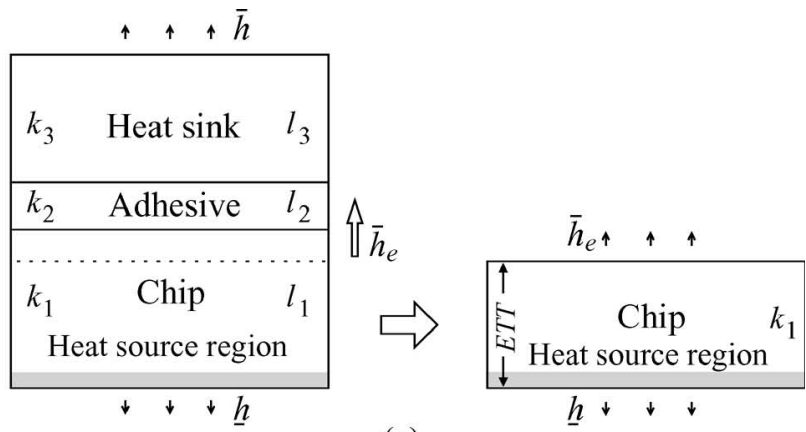

(a)

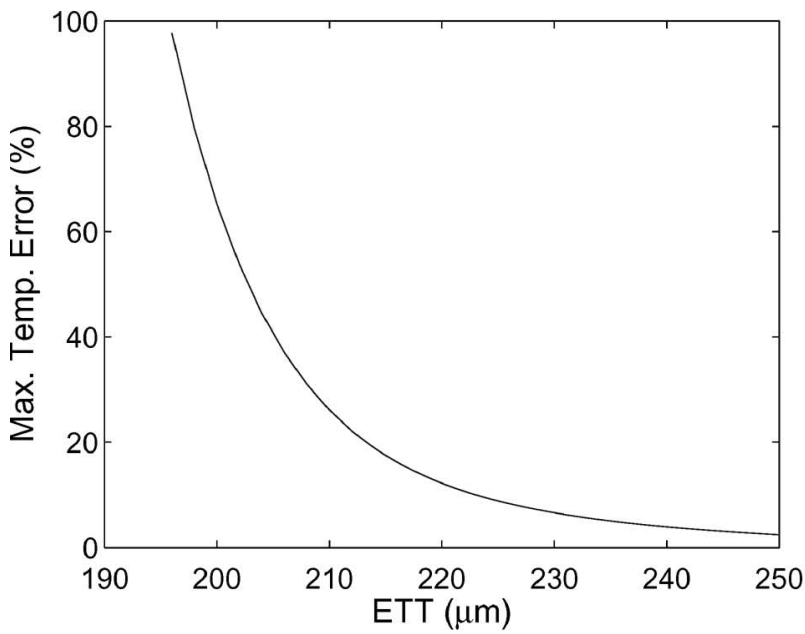

(b)

Fig. 11. STM and its accuracy versus the ETT. (a) MTM and its STM approximation (the rightmost diagram). (b) Accuracy of the STM versus ETT.

For comparisons, a simple matrix-vector product program was implemented to emulate the traditional Green's functionbased thermal-analysis methods [14]-[16]. The CPU usage of LOTAGre and the traditional methods is also shown in the table in Fig. 10. According to the table, when the number of cells $A \times B$ doubles, the temperature evaluation time by LOTAGre (LOTAGre) increased a little more than twofold while the run time by the traditional methods (Trad.) increased around four times. These run-time data closely match the theoretical complexities of LOTAGre and the traditional methods. LOTAGre clearly has superior computing speed and is also scalable to large problem size. For example, when $A \times B$ increased to $1024 \times 1024$, a chip of one million standard cells was analyzed in less than $8 \mathrm{~s}$ by LOTAGre. Contrastingly, the traditional methods became extremely slow even when the number of cells was no more than $256 \times 512$, or one-eighths of one million.

\section{STM and Effective Thermal Thickness (ETT)}

In the thermal analysis of a real multiple-layer structured IC, traditional Green's function-based methods usually model the heat-conduction path as a one-layer structure, by approximating the MTM by an STM with effective heat transfer rates to the ambient. Fig. 11(a) illustrates the STM approximation to the MTM of the example chip. In the figure, $\bar{h}_{\mathrm{e}}$ is the effective heat transfer rate from the top of the STM to the ambient, determined by the approach in [34]; and ETT is called the effective thermal thickness.

To determine the accuracy of the STM approximation for the example chip shown in Fig. 8, the STM in the rightmost diagram in Fig. 11(a) was used in LOTAGre to analyze the temperature distribution of the heat-source region. The temperatures obtained by using this STM approximation were compared with the temperatures generated by FLUENT simulation of the MTM in Fig. 8(b). The maximum percentage errors of the calculated temperatures based on the STM, versus the ETT, are plotted in Fig. 11(b). The figure shows that the accuracy of using the STM is very sensitive to the ETT. For the simulated chip, when ETT $=250 \mu \mathrm{m}$, the maximum percentage error of the calculated temperatures using the STM is $2.41 \%$; when ETT $=240 \mu \mathrm{m}$, the maximum percentage error is $3.93 \%$; however, when ETT $=210 \mu \mathrm{m}$, the percentage error is as large as $26.14 \%$. To ensure the temperature errors within $2.4 \%$, both the chip region and the thermal adhesive should be modeled. However, this is beyond the capability of the STM. When the STM is used for IC thermal analysis, the ETT should be determined very accurately, due to the large sensitivity of the accuracy of the STM to ETT. Compared to the STM approximation approach, LOTAGre is based on the MTM. Therefore, it can provide better accuracy and more modeling capability, in addition to have a low run-time complexity.

\section{CONCLUSiOn}

This paper has introduced a computationally efficient fullchip thermal-analysis method, called LOTAGre which is shown to have an $\mathcal{O}(N \lg N)$ complexity. The method solves the steady-state heat-conduction equation by mainly employing the multilayer heat-conduction Green's function in order to eliminate the computationally expensive modeling mandated by conventional numerical analysis methods such as FD and FE methods. Further, the proposed method, LOTAGre, analyzes the effects of ambient temperature on thermal distribution within a chip by solving the homogeneous solution based on general 2-D ambient temperature functions. By combining the eigenexpansion technique and classic TL theory, this paper has systematically derived analytical formulas for the homogeneous and inhomogeneous solutions to the heat-conduction equation, based on a general MTM. The MTM is capable of modeling multilayer ULSI chips with their accompanying heat sinks and mounting accessories. This paper considers different sidewall $\mathrm{BCs}$ and also gives fully analytical formulas for the $s$-domain multilayer Green's function. By conducting extensive amount of simulation experiments, this paper establishes that LOTAGre is as accurate as FLUENT, a commercial CFD tool. However, the use of the multilayer heat-conduction Green's function and DCT/IDCT algorithms enable LOTAGre to run two to three orders of magnitude faster than FLUENT and traditional Green's function-based methods. Finally, this paper discusses the empirical results of the conventionally used STM to point out its limitations in the thermal analysis of multiple-layer structured chips. 


\section{ACKNOWLEDGMENT}

The authors would like to thank the anonymous reviewers for their helpful suggestions and comments.

\section{REFERENCES}

[1] T. Sakurai and A. Newton, "Alpha-power law MOSFET model and its applications to CMOS inverter delay and other formulas," IEEE J. SolidState Circuits, vol. 25, no. 2, pp. 584-594, Apr. 1990.

[2] K. Kanda, K. Nose, H. Kawaguchi, and T. Sakurai, "Design impact of positive temperature dependence on drain currentin sub-1-V CMOS VLSIs," IEEE J. Solid-State Circuits, vol. 36, no. 10, pp. 1559-1564, Oct. 2001.

[3] C.-I. Kim, H. Soeleman, and K. Roy, "Ultra-low-power DLMS adaptive filter for hearing aid applications," IEEE Trans. Very Large Scale Integr. (VLSI) Syst., vol. 11, no. 6, pp. 1058-1067, Dec. 2003.

[4] B. Calhoun, A. Wang, and A. Chandrakasan, "Modeling and sizing for minimum energy operation in subthreshold circuits," IEEE J. Solid-State Circuits, vol. 40, no. 9, pp. 1778-1786, Sep. 2005.

[5] K. Banerjee, A. Amerasekera, G. Dixit, and C. Hu, "The effect of interconnect scaling and low- $k$ dielectric on the thermal characteristics of the IC metal," in IEDM Tech. Dig., Dec. 1996, pp. 65-68.

[6] D. Chen, E. Li, E. Rosenbaum, and S.-M. Kang, "Interconnect thermal modeling for accurate simulation of circuit timing and reliability," IEEE Trans. Comput.-Aided Design Integr. Circuits Syst., vol. 19, no. 2, pp. 197-205, Feb. 2000

[7] M. R. Casu, M. Graziano, G. Masera, G. Piccinini, M. M. Prono, and M. Zamboni, "Clock distribution network optimization under self-heating and timing constraints," in Proc. PATMOS, Sep. 2002, pp. 198-208.

[8] K. Banerjee, A. Mehrotra, A. Sangiovanni-Vincentelli, and C. Hu, "On thermal effects in deep sub-micron VLSI interconnects," in Proc. ACM/IEEE Des. Autom. Conf., Jun. 1999, pp. 885-891.

[9] M. Casu, M. Graziano, G. Masera, G. Piccinini, and M. Zamboni, "An electromigration and thermal model of power wires for a priori high-level reliability prediction," IEEE Trans. Very Large Scale Integr. (VLSI) Syst., vol. 12, no. 4, pp. 349-358, Apr. 2004.

[10] W. Huang, M. Stan, K. Skadron, K. Sankaranarayanan, S. Ghosh, and S. Velusamy, "Compact thermal modeling for temperature-aware design," in Proc. ACM/IEEE Des. Autom. Conf., Jun. 2004, pp. 878-883.

[11] L. Codecasa, D. D'Amore, and P. Maffezzoni, "An Arnoldi based thermal network reduction method for electro-thermal analysis," IEEE Trans. Compon. Packag. Technol., vol. 26, no. 1, pp. 186-192, Mar. 2003.

[12] T.-Y. Wang and C. Chen, "3-D thermal-ADI: A linear-time chip level transient thermal simulator," IEEE Trans. Comput.-Aided Design Integr. Circuits Syst., vol. 21, no. 12, pp. 1434-1445, Dec. 2002.

[13] A. Odabasioglu, M. Celik, and L. Pileggi, "PRIMA: Passive reducedorder interconnect macromodeling algorithm," IEEE Trans. Comput.Aided Design Integr. Circuits Syst., vol. 17, no. 8, pp. 645-654, Aug. 1998.

[14] Y.-K. Cheng and S.-M. Kang, "A temperature-aware simulation environment for reliable ULSI chip design," IEEE Trans. Comput.-Aided Design Integr. Circuits Syst., vol. 19, no. 10, pp. 1211-1220, Oct. 2000.

[15] B. Wang and P. Mazumder, "Fast thermal analysis for VLSI circuits via semi-analytical Green's function in multi-layer materials," in Proc. Int. Symp. Circuits and Syst., May 2004, vol. 2, pp. 409-412.

[16] Y. Zhan and S. Sapatnekar, "Fast computation of the temperature distribution in VLSI chips using the discrete cosine transform and table look-up," in Proc. Asia and South Pacific Des. Autom. Conf., Jan. 2005, vol. 1, pp. 87-92.

[17] J. V. Beck, K. Cole, and A. Haji-Sheikh, Heat Conduction Using Green's Functions. Bristol, PA: Hemisphere, 1992.

[18] K. Banerjee, S. Souri, P. Kapur, and K. Saraswat, "3-D ICs: A novel chip design for improving deep-submicrometer interconnect performance and systems-on-chip integration," Proc. IEEE, vol. 89, no. 5, pp. 602-633, May 2001

[19] W. Batty, C. Christoffersen, A. Panks, S. David, C. Snowden, and M. Steer, "Electrothermal CAD of power devices and circuits with fully physical time-dependent compact thermal modeling of complex nonlinear 3-D systems," IEEE Trans. Compon. Packag. Technol., vol. 24, no. 4, pp. 566-590, Dec. 2001.

[20] M. Rencz, V. Szekely, A. Poppe, and B. Courtois, "Inclusion of RC compact models of packages into board level thermal simulation tools," in Proc. 18th IEEE Semicond. Thermal Meas. and Manage. Symp., 2002 , pp. $71-76$.
[21] K. Nabors and J. White, "FastCap: A multipole accelerated 3-D capacitance extraction program," IEEE Trans. Comput.-Aided Design Integr. Circuits Syst., vol. 10, no. 11, pp. 1447-1459, Nov. 1991.

[22] T.-Y. Chou and Z. Cendes, "Capacitance calculation of IC packages using the finite element method and planes of symmetry," IEEE Trans. Comput.Aided Design Integr. Circuits Syst., vol. 13, no. 9, pp. 1159-1166, Sep. 1994.

[23] J. Phillips and J. White, "A precorrected-FFT method for electrostatic analysis of complicated 3-D structures," IEEE Trans. Comput.-Aided Design Integr. Circuits Syst., vol. 16, no. 10, pp. 1059-1072, Oct. 1997.

[24] J. Zhao, W. Dai, S. Kadur, and D. Long, "Efficient three-dimensional extraction based on static and full-wave layered Green's functions," in Proc. ACM/IEEE Des. Autom. Conf., Jun. 1998, pp. 224-229.

[25] A. Niknejad, R. Gharpurey, and R. Meyer, "Numerically stable Green function for modeling and analysis of substrate coupling in integrated circuits," IEEE Trans. Comput.-Aided Design Integr. Circuits Syst., vol. 17, no. 4, pp. 305-315, Apr. 1998.

[26] C. Xu, T. Fiez, and K. Mayaram, "On the numerical stability of Green's function for substrate coupling in integrated circuits," IEEE Trans. Comput.-Aided Design Integr. Circuits Syst., vol. 24, no. 4, pp. 653-658, Apr. 2005.

[27] A. Kokkas, "Thermal analysis of multiple-layer structures," IEEE Trans. Electron Devices, vol. ED-21, no. 11, pp. 674-681, Nov. 1974.

[28] J. Albers, "An exact recursion relation solution for the steady-state surface temperature of a general multilayer structure," IEEE Trans. Compon., Packag., Manuf. Technol. A, vol. 18, no. 1, pp. 31-38, Mar. 1995.

[29] A. Csendes, V. Szekely, and M. Rencz, "An efficient thermal simulation tool for ICs, microsystem elements and MCMs: The $\mu$ S-THERMANAL," Microelectron. J., vol. 29, no. 4/5, pp. 241-255, 1998.

[30] Y. C. Gerstenmaier and G. Wachutka, "Time dependent temperature fields calculated using eigenfunctions and eigenvalues of the heat conduction equation," Microelectron. J., vol. 32, no. 10, pp. 801-808, Oct. 2001.

[31] J.-M. Dorkel, P. Tounsi, and P. Leturcq, "Three-dimensional thermal modeling based on the two-port network theory for hybrid or monolithic integrated power circuits," IEEE Trans. Compon., Packag., Manuf. Technol. A, vol. 19, no. 4, pp. 501-507, Dec. 1996.

[32] D. Maillet, S. Andre, J. C. Batsale, A. Degiovanni, and C. Moyne, Thermal Quadrupoles: Solving the Heat Equation Through Integral Transforms. Hoboken, NJ: Wiley, 2000.

[33] Y. Gerstenmaier and G. Wachutka, "Transient temperature fields with general nonlinear boundary conditions in electronic systems," IEEE Trans. Compon. Packag. Technol., vol. 28, no. 1, pp. 23-33, Mar. 2005.

[34] Y.-K. Cheng, P. Raha, C.-C. Teng, E. Rosenbaum, and S.-M. Kang, "ILLIADS-T: An electrothermal timing simulator for temperaturesensitive reliability diagnosis of CMOS VLSI chips," IEEE Trans. Comput.-Aided Design Integr. Circuits Syst., vol. 17, no. 8, pp. 668-681, Aug. 1998.

[35] J. Clementi, J. McCreary, T. Niu, J. Palomaki, J. Varcoe, and G. Hill, "Flip-chip encapsulation on ceramic substrates," in Proc. Electron. Compon. and Technol. Conf., Jun. 1993, pp. 175-181.

[36] J. Parry, H. Rosten, and G. Kromann, "The development of componentlevel thermal compact models of a C4/CBGA interconnect technology: The Motorola PowerPC 603 and PowerPC 604 risc microprocessors," IEEE Trans. Compon., Packag., Manuf. Technol. A, vol. 21, no. 1, pp. 104-112, Mar. 1998.

[37] R. Crampagne, M. Ahmadpanah, and J.-L. Guiraud, "A simple method for determining the Green's function for a large class of MIC lines having multilayered dielectric structures," IEEE Trans. Microw. Theory Tech., vol. MTT-26, no. 2, pp. 82-87, Feb. 1978.

[38] J. Brown and R. Churchill, Fourier Series and Boundary Value Problems, 5th ed. New York: McGraw-Hill, 1993.

[39] R. H. Joseph, Microwave Theory and Techniques. Cambridge, MA: Boston Technical Publishers, 1965.

[40] D. M. Pozar, Microwave Engineering. Reading, MA: Addison-Wesley, 1990.

[41] V. Szekely, A. Poppe, M. Rencz, M. Rosental, and T. Teszeri, "THERMAN: A thermal simulation tool for IC chips, microstructures and PW boards," Microelectron. J., vol. 40, no. 3, pp. 517-524, Mar. 2000.

[42] V. Szekely, "THERMODEL: A tool for compact dynamic thermal model generation," Microelectron. J., vol. 29, no. 4, pp. 257-267, Apr. 1998.

[43] _ "Identification of RC networks by deconvolution: Chances and limits," IEEE Trans. Circuits Syst. I, Fundam. Theory Appl., vol. 45, no. 3, pp. 244-258, Mar. 1998.

[44] N. Ahmed, T. Natarjan, and K. R. Rao, "Discrete cosine transform," IEEE Trans. Comput., vol. C-23, no. 1, pp. 90-93, Jan. 1974.

[45] G. J. Miao and M. A. Clements, Digital Signal Processing and Statistical Classification. Norwood, MA: Artech, 2002. 


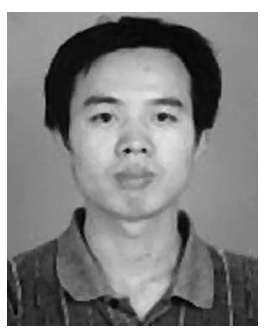

Baohua Wang received the M.S. degree in computer science from Tsinghua University, Beijing, China. $\mathrm{He}$ is currently working toward the Ph.D. degree in computer science and engineering at the University of Michigan, Ann Arbor.

His research interests include physical design algorithms, circuit simulation and optimization, and nanometer circuit design issues.

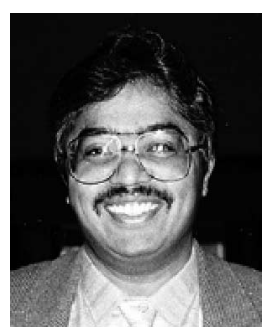

Pinaki Mazumder (S'84-M'88-SM'94-F'99) received the B.S.E.E. degree from the Indian Institute of Science, Bangalore, India, in 1976, the M.Sc. degree in computer science from University of Alberta, Edmonton, Alberta, Canada, in 1985, and the Ph.D. degree in electrical and computer engineering from University of Illinois at Urbana-Champaign, in 1987.

$\mathrm{He}$ is currently with the Department of Electrical Engineering and Computer Science, University of Michigan, Ann Arbor. He was a Research Assistant with the Coordinated Science Laboratory, University of Illinois at Urbana-Champaign for two years and was with the Bharat Electronics Ltd. (a collaborator of RCA), India, for over six years, where he developed several types of analog and digital integrated circuits for consumer electronics products. During the summer of 1985 and 1986, he was a member of the Technical Staff with the Indian Hill branch of AT\&T Bell Laboratories. During 1996 and 1997, he spent his sabbatical leave as a Visiting Faculty with Stanford University, University of California at Berkeley, and Nippon Telephone and Telegraph, Japan. His research interests include VLSI testing, physical design automation, and ultrafast circuit design. He has published over 160 papers on these topics in archival journals and proceedings of the international conferences. He has led his research group's efforts in VLSI testing and built-in self-repair techniques and has developed silicon compilers for RAM, ROM, and PLA with built-in self-repairable capabilities. Two international patents on this research work are pending now. He has also done quite extensive work in the area of VLSI physical design. He has developed a suite of distributed place-and-route tools for VLSI and FPGA chips. He has worked over six years as an Integrated Circuit Designer with semiconductor companies. He is currently leading ultrafast circuit design activities for nano and quantum electronic devices. He has successfully developed CAD tools for high-performance VLSI circuit simulation (NDR-SPICE) and numerous circuit topologies for quantum MOS and other quantum-well devices. Several U.S. and Japanese semiconductor companies including Texas Instruments, Hughes Research Laboratory, Lockheed Martin, NTT and NEC have been collaborating with him on this research work. He is the coauthor of Testing and Testable Design of High-Density RandomAccess Memories (Norwell, MA: Kluwer, 1996), Semiconductor RandomAccess Memories: Testing and Reliability (Rockville, MD: Computer Science), Genetic Algorithms for VLSI Design, Layout, and Test Automation (Englewood Cliffs, NJ: Prentice-Hall, 1998).

Dr. Mazumder was a recipient of Digital's Incentives for Excellence Award, BF Goodrich National Collegiate Invention Award, National Science Foundation Research Initiation Award, and Bell Northern Research Laboratory Faculty Award. He was a Guest Editor of the IEEE Design and Test Magazine's Special Issue on multimegabit memory testing, in 1993 and the Journal of Electronic Testing: Theory and Applications Special Issue on Memory Testing and Reliability in 1994. He is the Guest Editor of two special issues on emerging nanoelectronic technologies and their applications in IEEE TRANSACTIONS ON Very Large Scale InTEgration (VLSI) Systems (December 1997) and the ProceEdings OF THE IEEE (1998). He is on the Editorial Board of ProceEdings of THE IEEE and is also an Associate Editor of the IEEE Transactions on Very LaRge Scale Integration (VLSI) Systems. $\mathrm{He}$ is a member of Sigma Xi, Phi Kappa Phi, and ACM SIGDA. 